\title{
Altered sensory processing and dendritic remodeling in hyperexcitable visual cortical networks
}

\author{
Eleonora Vannini $^{1,2} \cdot$ Laura Restani $^{1} \cdot$ Marta Pietrasanta $^{1,2} \cdot$ Alessandro Panarese $^{1,3}$. \\ Alberto Mazzoni $^{3} \cdot$ Ornella Rossetto $^{4} \cdot$ Silvia Middei $^{5} \cdot$ Silvestro Micera ${ }^{3,6}$. \\ Matteo Caleo 1
}

Received: 17 February 2015/ Accepted: 1 July 2015

(C) Springer-Verlag Berlin Heidelberg 2015

\begin{abstract}
Epilepsy is characterized by impaired circuit function and a propensity for spontaneous seizures, but how plastic rearrangements within the epileptic focus trigger cortical dysfunction and hyperexcitability is only partly understood. Here we have examined alterations in sensory processing and the underlying biochemical and neuroanatomical changes in tetanus neurotoxin (TeNT)induced focal epilepsy in mouse visual cortex. We documented persistent epileptiform electrographic discharges and upregulation of GABAergic markers at the completion of TeNT effects. We also found a significant remodeling of the dendritic arbors of pyramidal neurons, with increased dendritic length and branching, and overall reduction in spine density but significant preservation of
\end{abstract}

Electronic supplementary material The online version of this article (doi:10.1007/s00429-015-1080-1) contains supplementary material, which is available to authorized users.

Laura Restani

restani@in.cnr.it

1 CNR Neuroscience Institute, via G. Moruzzi 1, 56124 Pisa, Italy

2 Scuola Normale Superiore, Pisa, Italy

3 The BioRobotics Institute, Scuola Superiore Sant'Anna, Pisa, Italy

4 Department of Biomedical Sciences, University of Padua, Padua, Italy

5 CNR Institute of Cell Biology and Neurobiology, Rome, Italy

6 Bertarelli Foundation Chair in Translational Neuroengineering, School of Engineering, Center for Neuroprosthetics and Institute of Bioengineering, École Polytechnique Fédérale de Lausanne (EPFL), Lausanne, Switzerland mushroom, mature spines. Functionally, spontaneous neuronal discharge was increased, visual responses were less reliable, and electrophysiological and behavioural visual acuity was consistently impaired in TeNT-injected mice. These data demonstrate robust, long-term remodeling of both inhibitory and excitatory circuitry associated with specific disturbances of network function in neocortical epilepsy.

Keywords Tetanus neurotoxin - Visual cortex $\cdot$ Dendritic spines $\cdot$ Spontaneous activity $\cdot$ Visual acuity $\cdot$ Epilepsy

\section{Introduction}

Epilepsy is characterized by recurrent spontaneous seizures and in about one third of the patients cannot be controlled adequately by conventional anticonvulsant therapy (Simonato et al. 2012). In lesional forms of focal epilepsy, such as mesial temporal lobe epilepsy, degeneration of interneurons and sprouting of pyramidal cells shift the balance of excitation and inhibition towards excitation (Dudek and Sutula 2007). In cases where no brain damage is evident (non-lesional epilepsy), propensity to spontaneous seizures is likely generated by altered synaptic function at excitatory and/or inhibitory terminals (Corradini et al. 2014; Farisello et al. 2013; Ferecsko et al. 2014). In particular, chronic epilepsy is thought to result from rearrangements in synaptic organization that leave permanent marks on cortical networks (Pitkanen et al. 2013). The same circuit rearrangements may lead to network dysfunction (i.e. cognitive deficits and impaired information processing) even during interictal phases (Grant 2005). However, the cellular changes leading to altered synaptic drive onto hyperexcitable cortical neurons 
and consequent circuit dysfunction are only partly understood.

Injection of minute amounts of tetanus neurotoxin (TeNT) into the brain induces a chronic epileptic focus (Jefferys and Walker 2006). TeNT is a metalloprotease that enters synaptic terminals and cleaves the synaptic vesicle protein VAMP/synaptobrevin, resulting in selective blockade of neurotransmitter release (Rossetto et al. 2013; Schiavo et al. 2000). TeNT-induced epilepsy has been characterized in great detail with intrahippocampal (e.g., Jiruska et al. 2010; Whittington and Jefferys 1994) and intracortical injections (Hagemann et al. 1999; Louis et al. 1990; Mainardi et al. 2012; Nilsen et al. 2005; Wykes et al. 2012). In particular, delivery of TeNT to the adult cortex results in refractory epilepsy with electrographic seizures persisting for several months (Nilsen et al. 2005). Thus, hyperexcitability of the cortical network clearly outlives TeNT proteolytic activity (Mainardi et al. 2012).

TeNT appears to be particularly effective on GABAergic inhibitory neurons with a relative sparing of excitatory transmitter release. Recently, Jefferys and colleagues (Ferecsko et al. 2014) have demonstrated that TeNT reduces spontaneous excitatory postsynaptic currents (EPSCs) to $38 \%$ of control, while almost completely abolishing both spontaneous and evoked inhibitory postsynaptic currents (IPSCs). Thus, during the time window of TeNT action, hyperexcitability may be explained by a dramatic loss of inhibition with relative preservation of excitatory synaptic transmission (Ferecsko et al. 2014). However, the mechanisms that maintain propensity to spontaneous seizures after the completion of TeNT action are incompletely understood.

Recently, we have described the general features of TeNT-induced epilepsy in mouse visual cortex (Mainardi et al. 2012). We found that electrographic seizure activity and interictal discharges persist even when the toxin has been cleared from the system. At the anatomical level, there was no evidence for neuronal loss and gliosis (Mainardi et al. 2012). These data prompt the search for synaptic changes that maintain the network in a persistently hyperexcitable state. In this context, the excellent knowledge of the neuroanatomical and functional features of visual cortex provides a strong foundation for the elucidation of epileptogenic mechanisms. Here, we used this model to determine the biochemical and morphological rearrangements that underlie long-term network hyperexcitability. We also performed a quantitative analysis of electrophysiological and behavioral visual responses in the TeNT-infused cortex to examine disturbances of baseline perceptual function within the seizure focus.

\section{Materials and methods}

\section{Animals and TeNT injections}

Adult (age $>$ postnatal day 60) C57BL/6J mice were used in this study. Animals were reared in a $12 \mathrm{~h} \mathrm{light/dark}$ cycle, with food and water available ad libitum. All experimental procedures conformed to the European Communities Council Directive $n^{\circ}$ 86/609/EEC and were approved by the Italian Ministry of Health. TeNT injections were performed as previously described (Mainardi et al. 2012). Briefly, injections were performed into the primary visual cortex, i.e. $0.0 \mathrm{~mm}$ anteroposterior and $2.7 \mathrm{~mm}$ lateral to the lambda suture. TeNT (Lubio, Lucerne, Switzerland; $300 \mathrm{~nL}$ total volume; 0.1-1 ng) in phosphate-buffered saline (PBS) containing $2 \%$ rat serum albumin (RSA) was injected at a cortical depth of $650 \mu \mathrm{m}$. Control animals received RSA alone.

After surgery, a glucose solution (5\% in saline) was subcutaneously administered and recovery of animals was carefully monitored. Paracetamol was added in drinking water for 3 days.

\section{Local field potential (LFP) recordings}

LFP recordings were performed as previously described (Mainardi et al. 2012). Briefly, bipolar recordings were collected by placing a couple of Nichrome wire electrodes to sample the local electrical activity originating in the TeNT-injected visual cortex. Under Hypnorm/Hypnovel anesthesia (1.4 ml/100 g body weight), mice were placed in a stereotaxic apparatus, and two electrodes were positioned epidurally (2.5 and $3.5 \mathrm{~mm}$ lateral and $0.0 \mathrm{~mm}$ anteroposterior to lambda); a ground screw was placed in the occipital bone. Recordings were performed at 10 $(n=2)$ and 45 days $(n=3)$ following TeNT injections. Animals were habituated for $1 \mathrm{~h}$ to the test cage before a $1 \mathrm{~h}$ recording session, using a digital acquisition system. Cortical LFP signals were acquired with a sampling rate of $200 \mathrm{~Hz}$ as the differential between the two adjacent electrode sites, 50,000X amplified and 0.3-100 Hz band-passed. Analysis of epileptiform activity was performed using a custom-made application, based on LabView (National Instruments), as described previously (Antonucci et al. 2009; Mainardi et al. 2012). The program first identified epileptiform alterations and spikes in the EEG using a voltage threshold, which was set to 3 times the standard deviation of the EEG signal (determined during periods of baseline activity). Spikes were grouped in clusters when they were spaced by less than $1.5 \mathrm{~s}$ and defined as seizures, when they lasted more than $4 \mathrm{~s}$ (Antonucci et al. 2009; Mainardi et al. 2012). Frequency of epileptiform events in 
the animals used in the present study was comparable to what reported previously (Mainardi et al. 2012).

\section{Western blot}

Western blot was performed as previously described (Baldini et al. 2013; Restani et al. 2011, 2012). Briefly, visual cortices were dissected and frozen on dry ice. Proteins were extracted with lysis buffer $(20 \mathrm{mM}$ Tris- $\mathrm{HCl}$, pH 7.45, 150 mM NaCl, 10 mM EDTA, 0.1 mM Na3VO4, $1 \mathrm{mM}$ PMSF, $1 \mathrm{~g} / \mathrm{ml}$ leupeptin, $1 \mathrm{~g} / \mathrm{ml}$ aprotinin, $1 \%$ Triton X-100, and $10 \%$ glycerol) and total concentration of the samples was assessed with a protein assay kit (BioRad) using a bovine serum albumin-based standard curve. Total proteins loaded per lane were $10 \mu \mathrm{g}$ to detect each target protein, $50 \mu \mathrm{g}$ were loaded only for cleavedVAMP2. Protein extracts of each sample were separated by electrophoresis and blotted; filters were blocked and incubated overnight at $4{ }^{\circ} \mathrm{C}$ with primary antibodies (antiVAMP2, mouse monoclonal, 1:10,000; anti vGAT, mouse monoclonal, 1:1000; anti- vGlut1, rabbit polyclonal, 1:5000; all from Synaptic Systems; anti-GAD65/67, rabbit polyclonal, 1:5000, Sigma-Aldrich; anti-vGlut2, mouse monoclonal, 1:500, Millipore; anti-SNAP25, mouse monoclonal, 1:1000, Covance; anti-cleaved VAMP2, rabbit polyclonal, 1:100). Filters were also probed with anti- $\alpha$ tubulin antibody (mouse monoclonal, Sigma-Aldrich, or rabbit polyclonal, Abcam; at 1:15,000 dilution) as an internal standard for protein quantification. Secondary antibodies conjugated with infrared-emitting dyes (antimouse IRDye 680LT at 1:30,000 or anti-rabbit $800 \mathrm{CW}$ at 1:20,000; Li-Cor Biosciences) were used. Filters were scanned using an Odyssey IR scanner (Li-Cor Biosciences), and densitometry analysis was performed with Image Studio software version 3.1 (Li-Cor Biosciences). Antibody signal was calculated as integrated intensity of the region defined around the band of interest.

\section{Immunohistochemistry}

Ten days after TeNT, mice were deeply anaesthetized with intraperitoneal injections of chloral hydrate $(12.5 \%$ solution; $4 \mathrm{~mL} / \mathrm{kg}$ ) and perfused through the heart with freshly prepared $4 \%$ paraformaldehyde in $0.1 \mathrm{M}$ phosphate buffer, $\mathrm{pH}$ 7.4. Immunohistochemistry was performed as previously described (Mainardi et al. 2012; Restani et al. 2011). Briefly, brains were dissected and postfixed for $2 \mathrm{~h}$ at $4{ }^{\circ} \mathrm{C}$. Brain sections (40 $\mu \mathrm{m}$ thick) were cut with a freezing microtome.

For immunostaining, sections were blocked with $10 \%$ normal donkey serum in PBS containing $0.5 \%$ Triton $\mathrm{X}-100$ and then incubated overnight at $4{ }^{\circ} \mathrm{C}$ with the mouse monoclonal anti-VAMP2 (1:500 dilution; Synaptic
Systems) or with the guinea-pig polyclonal anti-NeuN (1:1000, Millipore). On the following day, sections were rinsed and incubated for $2 \mathrm{~h}$ at room temperature in solutions containing secondary antibodies diluted 1:500, and conjugated with Rhodamine Red-X (Jackson ImmunoResearch Laboratories). Sections were washed in PBS and mounted using an anti-fading agent (Vectashield; Vector Laboratories).

Sections were scanned with a microscope (Axio Imager.Z2, Zeiss) equipped with Apotome.2 (Zeiss) at resolutions of $1350 \times 1024$ pixels. Images were obtained using a $10 \times$ EC-PLAN-NEOFLUAR objective (NA 0.3) or a $40 \times$ EC-PLAN-NEOFLUAR oil objective (NA 0.75).

\section{Extracellular recordings in anesthetized mice}

Recordings were performed as described previously (Gianfranceschi et al. 2003; Pinto et al. 2009). Mice were anesthetized with urethane $(7 \mathrm{ml} / \mathrm{kg} ; 20 \%$ solution in saline, i.p.; Sigma) and placed in a stereotaxic apparatus. Analysis of epileptiform alterations was not performed in these animals due to the well-known effects of urethane anesthesia on EEG activity (e.g. Avoli and de Curtis 2011; Friedberg et al. 1999). Body temperature during the experiments was constantly monitored with a rectal probe and maintained at $37{ }^{\circ} \mathrm{C}$ with a heating blanket. The electrocardiogram was also continuously monitored. The depth of anesthesia was evaluated by pinch withdrawal reflex and other physical signs (respiratory and heart rate), as well as by observation of the EEG patterns and their dominant frequencies (after Fourier analysis of the signals; Caleo et al. 2003; Friedberg et al. 1999). A portion of the skull overlying the occipital cortex was drilled on one side, and a tungsten electrode (FHC; $1 \mathrm{M} \Omega$ ) was mounted on a three-axis motorized micromanipulator and inserted into the portion of visual cortex previously injected with TeNT or vehicle solution (coordinates: $3-3.3 \mathrm{~mm}$ lateral and in correspondence with lambda). Three penetrations were performed per animal. Visual evoked potentials (VEPs) were recorded at a depth of 100-150 $\mu \mathrm{m}$ within the cortex. The multiunit spiking activity was also recorded mainly from layers II-III and IV (i.e., within a depth of $400-500 \mu \mathrm{m}$ from the cortical surface).

\section{Visual stimuli and data analysis}

All visual stimuli were computer-generated on a display (Sony; $40 \times 30 \mathrm{~cm}$; mean luminance $15 \mathrm{~cd} / \mathrm{m}^{2}$ ) by a VSG card (Cambridge Research Systems).

Transient VEPs were recorded in response to abrupt reversal $(1 \mathrm{~Hz})$ of a horizontal square wave grating (spatial frequency, $0.06 \mathrm{c} / \mathrm{deg}$; contrast $90 \%)$. Signals were amplified (5000-fold), band pass filtered $(0.5-100 \mathrm{~Hz})$, and 
fed into a computer for storage and analysis. At least 30 events were averaged in synchrony with the stimulus contrast reversal. The response to a blank stimulus $(0 \%$ contrast) was also frequently recorded to estimate noise. Transient VEP responses were evaluated by measuring the peak to through amplitude of the major positive component.

Steady-state VEPs were recorded in response to reversal (4 Hz) of a horizontal sinusoidal grating of different spatial frequencies and contrast. For each stimulus, at least 100 events were averaged in synchrony with the contrast reversal. VEP amplitude was quantified by measuring the amplitude of the second harmonic of the Fourier transform computed from the recorded signal, as previously described (Caleo et al. 2003; Ferrari et al. 2013; Pietrasanta et al. 2014). The response to a blank ( $0 \%$ contrast) stimulus was also frequently recorded to estimate noise. For signal-tonoise analysis, we took the mean VEP amplitude evoked by stimulation with an optimal stimulus grating $(0.06 \mathrm{c} / \mathrm{deg}$, $90 \%$ contrast, $4 \mathrm{~Hz}$ ) divided by the average noise level (blank stimulus). Visual acuity was assessed after presentation of gratings of variable spatial frequencies $(90 \%$ contrast) and was taken as the highest spatial frequency that evoked a VEP response greater than the mean value of the noise.

In each animal, we also evaluated the contrast gain function (contrast 10, 20, 30, $90 \%$ ) in response to $0.06 \mathrm{c} /$ deg gratings. We fitted offline the contrast response function of each mouse with a standard hyperbolic ratio function (Albrecht and Hamilton 1982):

$\left[R C=R_{0}+R_{\max } \cdot C_{n} C_{50 n}+C_{n}\right]$

where $C$ is the contrast level and the response $R$ is given by the VEP amplitude. We took into account the effects of baseline activity and total responsiveness by estimating $R_{0}$ and $R_{\max }$ as the average over trials of the response at minimum and maximum tested contrast (Contreras and Palmer 2003). We then estimated the sensitivity parameters C50 and $n$ with the nlinfit function in Matlab Statistics Toolbox (Mathworks, Natick, MA), which finds optimal fit values using the Levenberg-Marquard algorithms to minimize squared errors (Contreras and Palmer 2003). We finally compared the parameters distribution in sessions with TeNT- and Vehicle- injected mice (Vaiceliunaite et al. 2013) to assess whether the animals in the two conditions displayed different contrast sensitivity.

For recordings of spiking activity, the visual stimulus consisted of a light bar (contrast, $100 \%$; thickness, $3^{\circ}$; speed, $28 \% \mathrm{sec}$ ) drifting in the central part of the visual field. Signals were amplified 25,000-fold and bandpass filtered (500-5000 Hz). Spikes were discriminated from background by a voltage threshold, which was set as 4.5 times the standard deviation of noise, as described previously (Cerri et al. 2010; Resta et al. 2007; Restani et al. 2009). Peak responses were determined from peristimulus time histograms (bin size $=33 \mathrm{~ms}$ ), averaged over 15 stimulus presentations. Drifting light bars were presented interleaved with blank-screen stimuli to evaluate spontaneous discharge. Pre-stimulus activity was estimated as the average firing rate (spikes/sec) measured in $1 \mathrm{~s}$-intervals before stimulus onset.

We also measured the percentage of failures, i.e. the proportion of stimulus presentations unable to elicit a significant modification of spiking activity in TeNT- and vehicle treated mice. A trial was considered as a failure when the visually-driven response was neither aligned nor comparable in magnitude with the average response (i.e., at least two-fold lower than the average peak discharge during 15 bar passages).

All analysis of electrophysiological data was performed blind to animal treatment.

\section{Behavioral assessment of visual acuity}

Behavioral assessment of visual acuity was performed starting from 45 days following TeNT or vehicle injections. Mice were tested, blind to treatment, in the visual water task as described previously in rodents (Baroncelli et al. 2012; Caleo et al. 2007; Pizzorusso et al. 2006; Prusky et al. 2000). Briefly, the device consisted of a trapezoidal-shaped pool, filled with water, partially divided at one end in two arms by a divider. Visual stimuli consisted of gratings of various spatial frequencies or gray fields, which were generated with two computer monitors placed at the end of each arm. A hidden platform was placed below the grating. Animals were released from the undivided end of the box and trained to associate the stimulus grating with the submerged platform (i.e., escape from water). The position of the grating and the platform was alternated in a pseudorandom sequence. The visual water task trains animals to distinguish initially a low $(0.1 \mathrm{c} / \mathrm{deg})$ spatial frequency vertical grating from gray and then tests the limit of this ability at higher spatial frequencies. The limit of discrimination was estimated by increasing the spatial frequency of the grating until performance fell below $70 \%$ accuracy (Prusky et al. 2000).

\section{Golgi-Cox staining}

Mice were deeply anaesthetized with chloral hydrate $(500 \mathrm{mg} / \mathrm{kg}$ i.p.) and perfused transcardially with $0.9 \%$ saline solution. After perfusion, brains were dissected and immediately immersed in a Golgi-Cox solution (potassium dichromate $5 \%$, mercuric chloride $5 \%$ and potassium chromate $5 \%$ ) and kept in the dark for 6 days at RT. On the seventh day, brains were transferred into a $30 \%$ 
sucrose solution (Gibb and Kolb 1998). Coronal sections (200 $\mu \mathrm{m}$ thickness) were cut with a vibratome (reservoir was filled with $6 \%$ sucrose and blade prepared for sectioning by immersion in xylene for $5 \mathrm{~min}$ ). Slices were immediately collected on $2 \%$ gelatin-coated microscope slides. Golgi staining was developed in the dark at RT as follows: sections were washed in distilled $\mathrm{H}_{2} \mathrm{O}$, incubated in ammonium hydroxide (Sigma-Aldrich) for $30 \mathrm{~min}$, dipped in Kodak Fix solution (Rapid fixer; Sigma-Aldrich) for $30 \mathrm{~min}$, washed in distilled $\mathrm{H}_{2} \mathrm{O}$, dehydrated, sunk in solution $\mathrm{x}(1 / 3$ chloroform, 1/3 xylene, 1/3 Absolute ethanol) for $15 \mathrm{~min}$ and in xylene for $15 \mathrm{~min}$. Sections were then mounted with Eukitt Fluka (Sigma).

\section{Dendritic branching and spine density analysis}

The Golgi-impregnated neocortical neurons were analyzed at high magnification (100X oil immersion objective) with the Neurolucida software (MicroBrightField Inc., Williston, USA) and a Zeiss microscope equipped with a motorized stage interfaced to a computer. We analyzed neurons located in the superficial layers of primary visual cortex, i.e. within the area covered by TeNT injection and sampled in our electrophysiological experiments. To select pyramidal neurons in visual cortex the following criteria were used: (i) characteristic triangular soma shape; (ii) full impregnation of the neurons with no apparent dendritic truncation; (iii) presence of at least two primary basilar and one apical dendrites, each of which branched at least once, and numerous dendritic spines (Colciaghi et al. 2014). Only protrusions with a clear connection of the head of the spine to the shaft of the dendrite were counted as spines using the Neurolucida software. The analysis was restricted to spines arising from basal dendrites. We used common conventions of spine subtype classification (Marchetti et al. 2010; Sorra and Harris 2000), and classified our spine sample into three distinct morphological classes (mushroom, thin and stubby). Number of spines/neurons per each group were as follows: Veh, 2739/45 from 9 animals; TeNT 10 days, 1191/23 from 4 animals; TeNT 45 days, 2642/35 from 5 animals.

A total of 25 neurons from vehicle-injected or naïve mice, and 30 neurons from TeNT-injected mice (10 and 20 cells at 10 and 45 days, respectively) were fully reconstructed three-dimensionally. These numbers are in line with previous publications using Golgi staining (Colciaghi et al. 2014; Marchetti et al. 2010) and are sufficient to yield a power greater than 0.90 in statistical comparisons. Dendrites arising from the cell body were considered as first-order until they bifurcated into secondorder segments and so on. The following parameters were quantified for each reconstructed neuron with the software program NeuroLucidaExplorer (MicroBrightField Inc.): (i) total dendritic length; (ii) dendritic branch number;(iii) total dendritic spine density (expressed as the ratio number of spines/length of dendritic segment, except first order ones), (iv) percentage of different morphologies for all the dendritic spines. For each neuron, the dendritic tree complexity was also quantified using Sholl analysis as follows: a transparent grid with concentric rings $10 \mu \mathrm{m}$ apart was automatically placed by the software over the dendritic drawings and the number of ring intersections was used to estimate the total dendritic length and arborization. The number of dendritic intersections crossing each $10 \mu \mathrm{m}$-radius circle progressively more distal from the soma was automatically counted. For each of these parameters, an average was calculated for each neuron. An investigator blind to the experimental condition carried out all analyses.

\section{Statistical analysis}

Statistical analysis was performed with SigmaPlot (version 12; Systat Software Inc., San José, CA, USA). Differences between two groups were assessed with Student's $t$ test when the observed treatment effects were normally distributed, or with the Mann-Whitney rank sum test when the samples were not drawn from normally distributed populations with the same variances. Differences between three or more groups were evaluated with one-way ANOVA, followed by a Tukey's or Holm-Sidak test for data normally distributed and with Kruskal-Wallis one-way ANOVA with Dunn's post hoc test for data non-normally distributed. Normality of distributions was assessed with Kolmogorov-Smirnov test.

The number of intersections in the Sholl analysis was examined with two-way ANOVA, followed by Holm-Sidak test. For contrast response curves, data were analysed by two-way repeated measures ANOVA followed by Holm-Sidak test, as a series of stimuli at different contrasts were presented to the same animal. Level of significance was $p<0.05$.

\section{Results}

\section{Persistent reduction in VAMP2 expression and hyperexcitability in TeNT-infused mice}

We initially used Western blotting to determine the time course of TeNT proteolytic action in the adult mouse visual cortex. In keeping with our previous report (Mainardi et al. 2012). TeNT-truncated VAMP2 peaked at 10 days and was completely extinguished 45 days after injection (Suppl. Figure 1a). At 10 days, we used immunostaining to assess the regional extension of TeNT 
proteolytic activity. We employed an antibody directed against the intact form of VAMP2 and we showed a strong reduction in VAMP2 levels throughout the injected primary visual cortex (Fig. 1a). We also analyzed whether this decrease in VAMP2 level may induce neurotoxicity at the peak of TeNT action. Immunohistochemistry for the specific neuronal marker NeuN showed similar cortical layering and neuronal structure in TeNT- and vehicleinjected animals (Suppl. Figure 1b). This is consistent with our previous neuronal counts and analysis of microglial activation showing lack of neuropathological signs following TeNT (Mainardi et al. 2012).

\section{a}
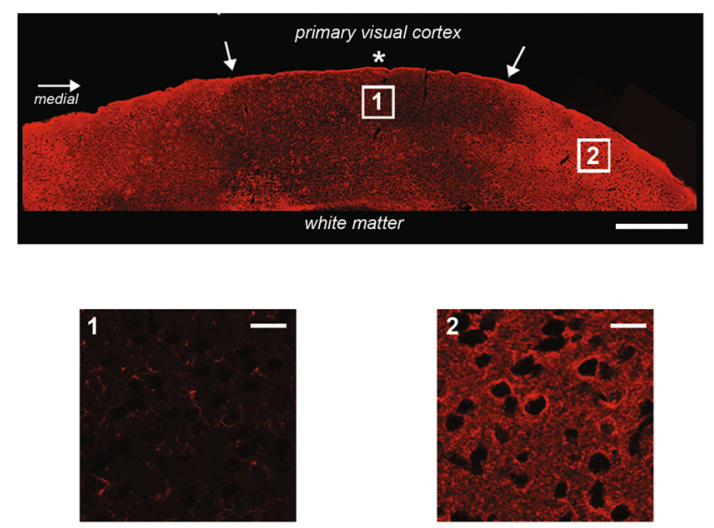

C
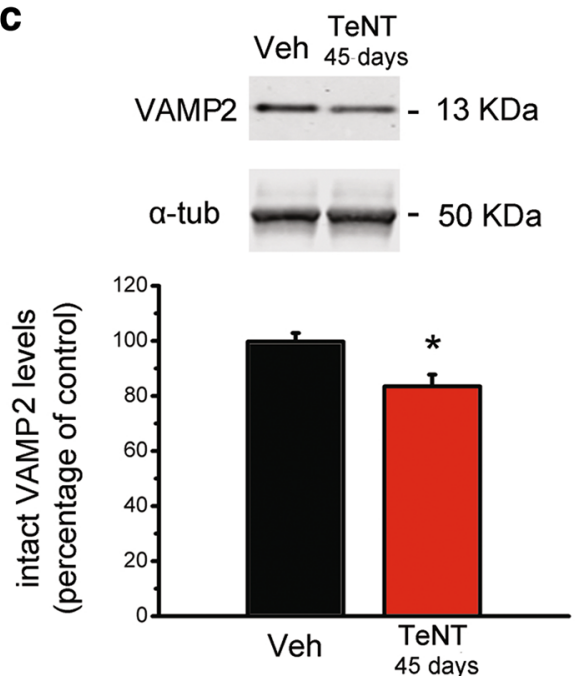

Fig. 1 Spread of TeNT action and persistent decrease of VAMP2 in the visual cortex. a Immunostaining for intact VAMP2 in a coronal section through the occipital cortex of an adult mouse, 10 days after TeNT injection. Note loss of VAMP2 labeling in the region corresponding to primary visual cortex (delimited by arrows), with respect to the medial and lateral areas (red staining). Scale bar $500 \mu \mathrm{m}$. Insets $(1,2)$ are magnifications of central $(1$, left $)$ and medial ( 2 , right) portion of the injected cortex. The asterisk indicates the injection site. Scale bar $10 \mu \mathrm{m}$. b Levels of intact VAMP2 at different
We also followed longitudinally the expression of the intact form of VAMP2 (Fig. 1b) and we found that VAMP2 levels were maximally reduced at 10-21 days with respect to naïve, uninjected mice. This was followed by a recovery that however remained incomplete (about $80 \%$ of control) at days 45-60 (one-way ANOVA followed by Holm-Sidak test, $p<0.009$; Fig. 1b). Thus, the reduction in intact VAMP2 appears to outlast the duration of TeNT proteolytic activity. These data were further confirmed by a quantitative comparison of Western blot signals from visual cortex samples of vehicle- and TeNTtreated mice 45 days after injection (Fig. 1c). Indeed, we

\section{b}

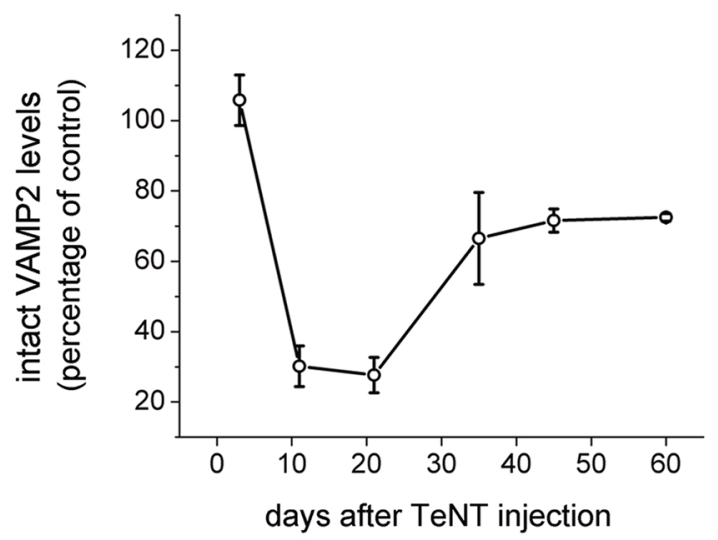

d
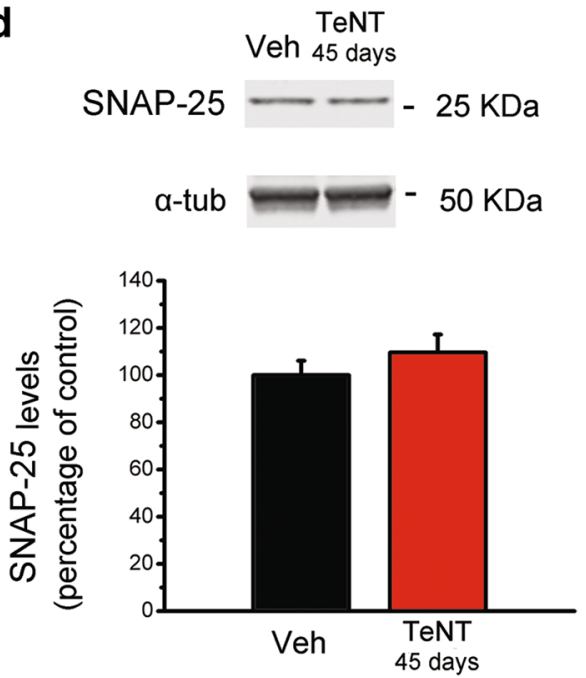

times after one single toxin injection. Note incomplete recovery of intact VAMP2 at the completion of TeNT effects (45-60 days; oneway ANOVA followed by Holm-Sidak test, $p<0.009$ ). For each time point, $n=4-8$ mice. c Comparison of intact VAMP2 expression in vehicle- and TeNT-infused mice at 45 days. Note reduced VAMP2 levels in TeNT samples $(t$ test, $p<0.012) n=7-9$ mice. ${ }^{*} p<0.05$. d Expression of SNAP-25 in vehicle- and TeNT-infused mice at 45 days. No differences are detectable between the two groups ( $t$ test, $p=0.35) ; n=5-6$ mice 
found that VAMP2 expression was significantly reduced in TeNT-treated animals as compared to controls injected with vehicle solution ( $t$ test, $p<0.012$ ). Another SNARE protein, synaptosomal associated protein of $25 \mathrm{kDa}$ (SNAP-25) showed no significant variation in TeNT-infused mice ( $t$ test, $p=0.35$; Fig. 1d), highlighting the specificity of the effect.

A subset of mice treated with the same TeNT batch were implanted with chronic bipolar electrodes for LFP recordings and evaluation of spontaneous epileptiform activity. In keeping with our recent report (Mainardi et al. 2012), interictal spiking and seizure activity were evident in the TeNT-treated cortex at both 10 and 45 days after injection (Suppl. Figure 1c). Behavioral seizures (e.g. twitches typically observed after TeNT delivery to the motor cortex; Nilsen et al. 2005) were not detected in TeNT animals, consistent with the fact that cleavage of VAMP2 remains spatially restricted to primary visual cortex (Fig. 1a) and does not spread to adjacent cortical areas (Mainardi et al. 2012). These data confirm that TeNT leads to the establishment of a chronic epileptic focus following the completion of toxin effects.

\section{Upregulation of GABAergic markers within the epileptic focus}

We next wondered whether specific alterations in either inhibitory or excitatory markers are associated to the epileptic phenotype of toxin-infused mice. To this aim, we measured by Western blot the expression of a battery of glutamatergic and GABAergic proteins in vehicle- and TeNT-treated mice at 10 and 45 days. We found no differences in the expression of vesicular glutamate transporters 1 and 2 (Fig. 2a, b; one way ANOVA, $p>0.5$ for both comparisons) that label intracortical and thalamocortical excitatory synapses, respectively (Mainardi et al. 2010; Nahmani and Erisir 2005). On the contrary, we found a significant increase of both the 65 and $67 \mathrm{kDa}$ isoforms of the GABA biosynthetic enzyme GAD (glutamate decarboxylase) in TeNT-injected mice at 45, but not 10 days (Fig. 2c; one way ANOVA followed by Dunn's test, $p<0.05$ for both isoforms). The vesicular GABA transporter vGAT also showed a trend for increased expression in TeNT-injected animals at 45 days (Fig. 2d; one way ANOVA, $p=0.22$ ). Thus, the GABA biosynthetic enzymes are significantly upregulated in the chronically epileptic cortex.

\section{Changes in dendritic structure and spine densities in the epileptic cortex}

To investigate structural underpinnings of the epileptic phenotype, we carried out a morphological reconstruction of Golgi-stained neurons in visual cortex (Fig. 3a, b) and quantified total dendritic length, dendritic complexity and spine densities. We focused on pyramidal neurons of superficial layers since these have been shown to be most sensitive to plasticity-inducing paradigms in the adult visual cortex (Goel and Lee 2007). We found that total dendritic length was similar in control (Veh) and TeNTinjected mice at 10 days, but significantly increased by about one-third in TeNT-treated mice at 45 days (one-way ANOVA followed by Dunn's test, $p<0.05$; Fig. 3c). Dendrite complexity was examined by Sholl analysis and the results showed only minor variations between controls and epileptic mice at 10 days (Fig. 3d). In contrast, robust differences were apparent between control and TeNTtreated neurons at 45 days. Indeed, we detected significantly increased numbers of intersections between dendrites and Sholl circles at a distance of 80-110 $\mu \mathrm{m}$ from the cell soma in the TeNT-treated group at 45 days (two-way ANOVA, $p<0.001$; post hoc Holm-Sidak test, $p<0.05$; Fig. 3d). Thus, overall dendritic length and dendritic complexity are consistently increased in the chronically epileptic cortex.

We also examined density and morphology of spines on basal dendrites of cortical pyramidal neurons (Fig. 4a). We measured spine density in the second, third and fourth branch order and we found a consistent spine loss in TeNTinfused animals, either at 10 or 45 days post-treatment (one-way ANOVA on Ranks followed by Dunn's test, $p<0.05$ for TeNT 10 days and $p<0.01$ for TeNT 45 days; Fig. 4b). Interestingly, in epileptic animals at either timepoint there was a significant increase in the proportion of mature and stable mushroom spines, and a corresponding decrease in the proportion of less mature, thin and stubby spines (Fig. 4c, one-way ANOVA, $p<0.01$; followed by post hoc test: $p<0.01$ for mushroom; $p=0.02$ for thin; $p=0.02$ for stubby). We also calculated the density of each spine subtype in the different animal groups. Consistent with the greater proportion of mushroom spines in epileptic animals, there was no significant reduction in the density of this morphological type of spine in TeNT-injected mice (one-way ANOVA on ranks, $p=0.145$; data not shown). These findings show an overall loss of dendritic protrusions with selective sparing of mushroom (i.e., mature) spines in epileptic animals.

\section{Persistent modifications of spontaneous discharge and visual responsiveness in epileptic mice}

To evaluate functional changes induced by TeNT injection, we performed extracellular spiking and field potential recordings from the visual cortex of anesthetized mice at 10 and 45 days after toxin (or vehicle) delivery. Analysis of multiunit activity revealed a profound enhancement of 
a
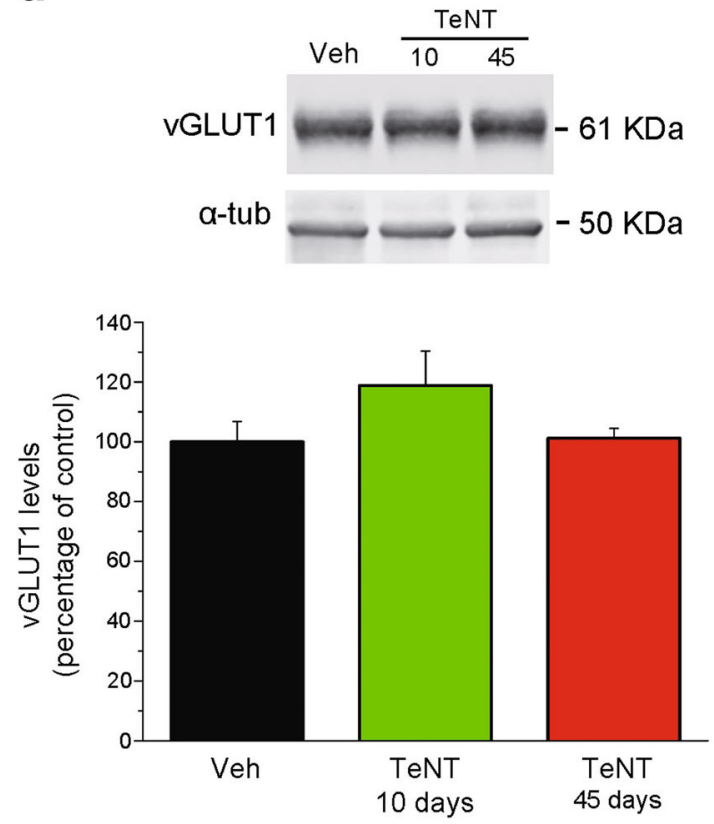

C
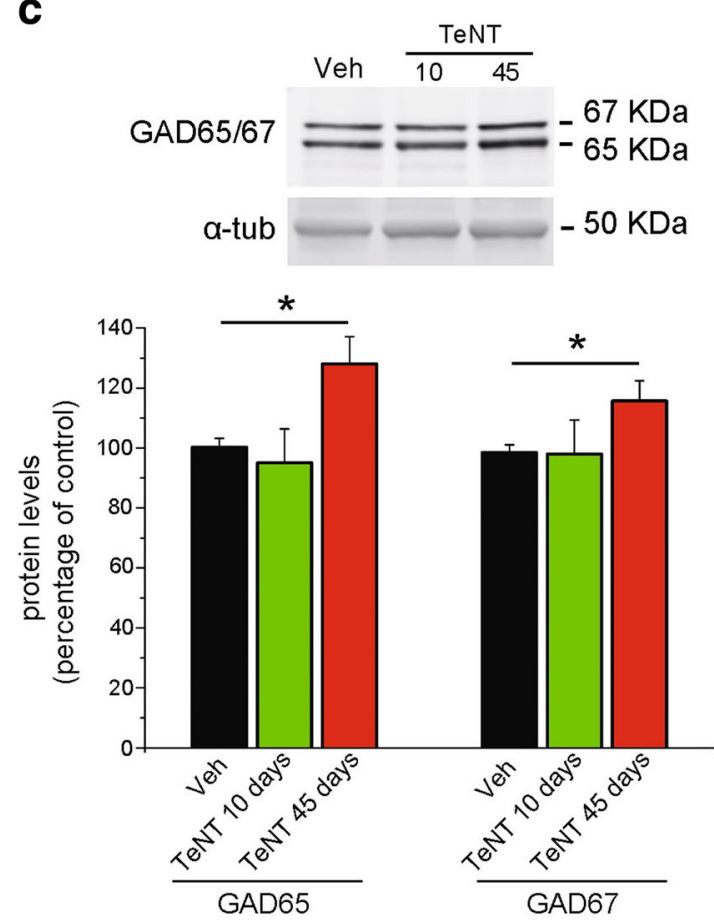

Fig. 2 Altered levels of the GABAergic markers in the visual cortex of TeNT-infused mice. Western blot analysis of cortical glutamatergic and GABAergic marker levels in vehicle and TeNT-treated mice, 10 and 45 days following injection. In all panels, the inset is a representative immunoblotting showing the corresponding protein levels in the cortex of animals in the three experimental groups (vehicle, TeNT 10 and 45 days). Expression of the presynaptic excitatory markers vGlut-1 (a) and vGlut-2 (b) in vehicle- and TeNTinjected mice. There is no significant difference between the two b
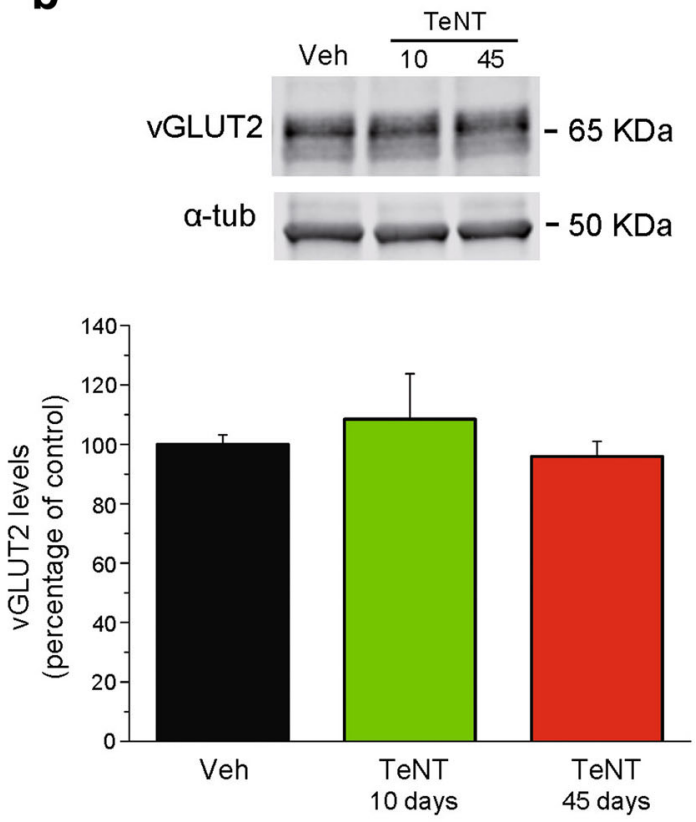

d
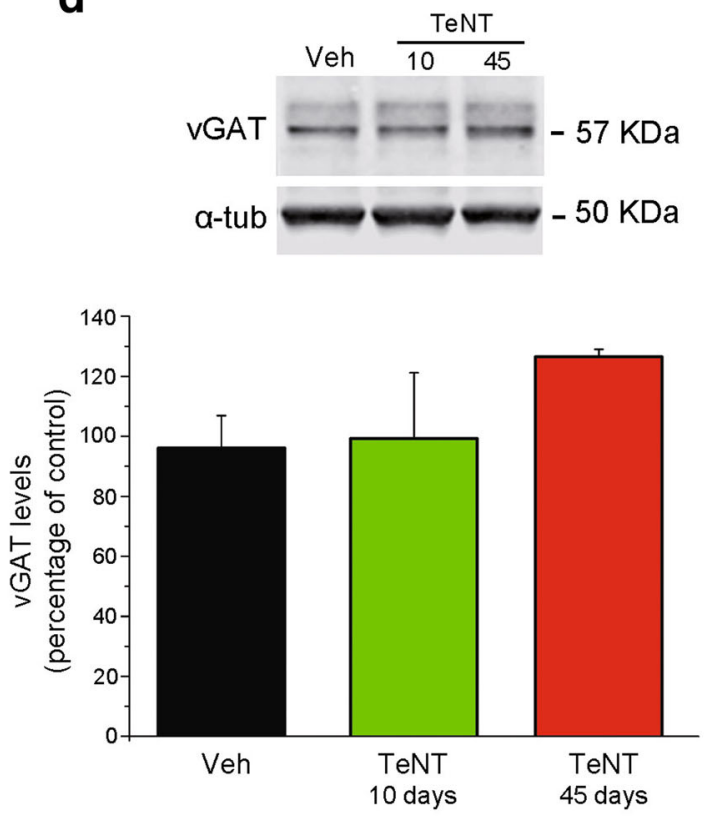

groups (one way ANOVA, $p>0.5$ for both markers). Expression of the GABAergic markers GAD65/67 (c) and vGAT (d) in vehicle- and TeNT-injected mice. The statistical analysis indicates consistent increases in GAD65/67 (one way ANOVA followed by Dunn's test, $p<0.05)$ in chronically epileptic animals. vGAT levels were increased at 45 days but this trend only approached statistical significance (one way ANOVA, $p=0.22$ ). For each histogram $n=5-10$ mice. ${ }^{*} p<0.05$. Data are mean \pm SEM 
a

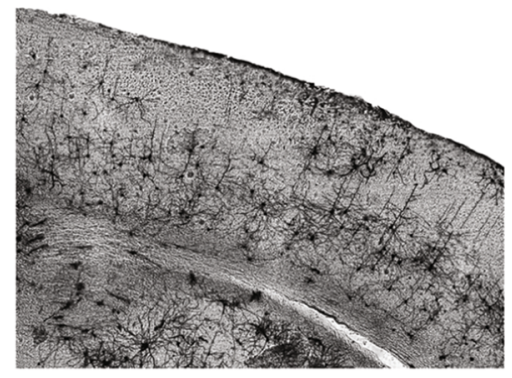

b

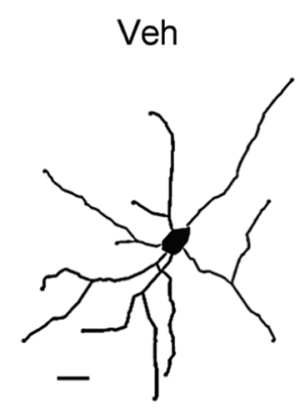

TeNT 10 days

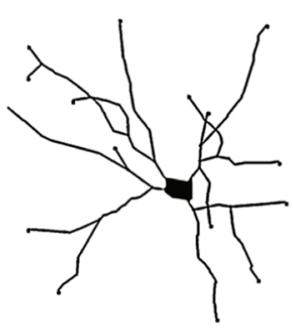

TeNT 45 days

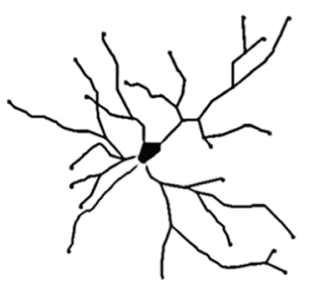

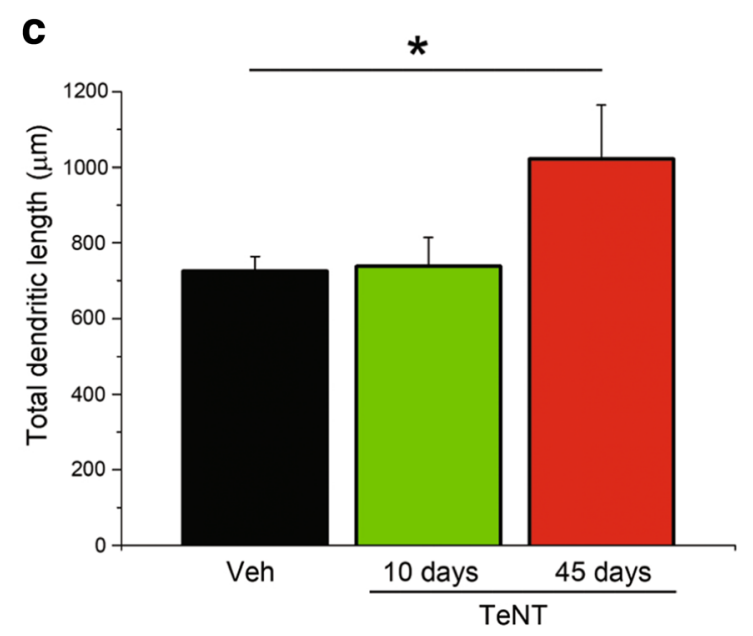

Fig. 3 Increased dendritic branching in epileptic mice. a Representative image of Golgi-impregnated cells in a coronal section through the mouse primary visual cortex. Scale bar $400 \mu \mathrm{m}$. b Neurolucida tracings of Golgi-stained cortical pyramidal neurons in vehicleinfused (Veh) and TeNT-injected animals at 10 and 45 days. Note the increase in total dendritic length in the epileptic cortex 45 days after TeNT. Scale bar $10 \mu \mathrm{m}$. c Quantitative analysis of total dendritic length reveals a statistically significant increase at 45 days, but not

spontaneous firing of cortical neurons in TeNT-treated mice at 10 days (Fig. 5a; one-way ANOVA on ranks, $p<0.001$; followed by Dunn's test, $p<0.01)$. This enhancement persisted at 45 days, i.e. when TeNT effects are cleared from the system (Fig. 5a; one-way ANOVA on ranks, $p<0.001$; followed by Dunn's test, $p<0.01$ ). We also quantified the peak response of cortical neurons to visual stimulation, i.e. the passage of an optimally oriented light bar within the receptive field. We found a profound depression of visually-driven discharges at 10 days (likely due to ongoing TeNT-mediated interference with synaptic transmission), but peak firing rates recovered at 45 days (one-way ANOVA on ranks, $p<0.001$; followed by Dunn's test $p<0.01$ for TeNT 10 days; Fig. 5b). We also computed cell responsiveness as the peak-to-baseline ratio, i.e. the peak discharge divided by spontaneous activity and we observed a very significant drop in visual d

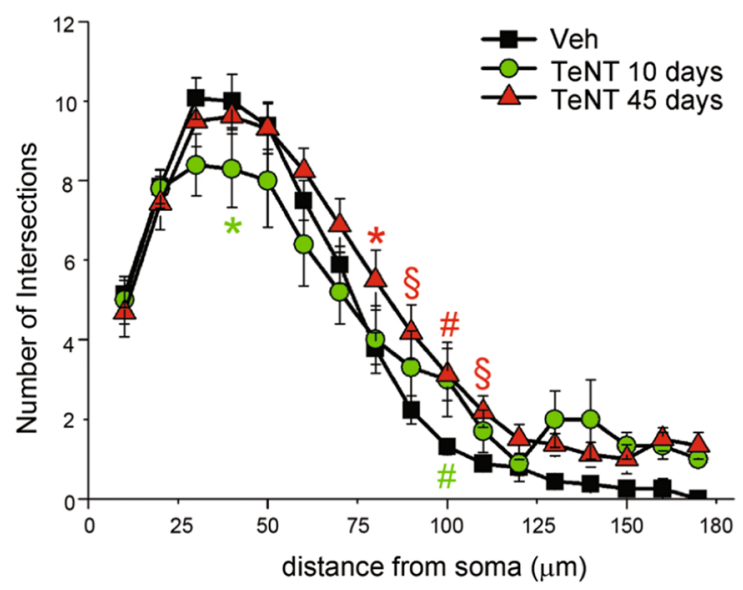

10 days, following TeNT delivery (one-way ANOVA, $p<0.05$; followed by Dunn's test, $p<0.05$ ). d Sholl analysis of cortical neurons in vehicle- and TeNT-infused mice, 10 and 45 days following delivery. Symbols indicate statistically significant differences across the dendritic trees between TeNT animals and vehicle controls (two-way ANOVA, $p<0.001$; followed by Holm Sidak test). ${ }^{*} p<0.05 ;{ }^{\#} p<0.01,{ }^{\S} p<0.001$. Data are mean \pm SEM

responsiveness at the peak of toxin activity (10 days), with significant but incomplete recovery at 45 days (one-way ANOVA on ranks, $p<0.001$; followed by Dunn's test, $p<0.01$ for TeNT 10 days and $p<0.05$ for TeNT 45 days, Fig. $5 c$ ).

The decrease in peak-to-baseline ratio in TeNT could be due to a decrease in the number of responsive units, in the sensitivity of responsive units, or both. To investigate whether the overall fraction of responsive units differed between vehicle and TeNT animals at 45 days, we computed the percent frequency distribution of the responsiveness (defined as the percentage of stimuli that elicited a response peak higher than mean $+2 \mathrm{SD}$ of the average baseline activity in the same unit) in the two animal groups. We found that the fraction of units with a responsiveness less than $30 \%$ increased from $9.3 \%$ in vehicle to $29.7 \%$ in TeNT, while the fraction of units with a responsiveness 
a

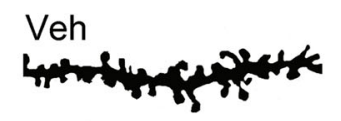

TeNT 10 days

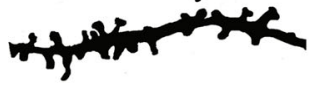

TeNT 45 days
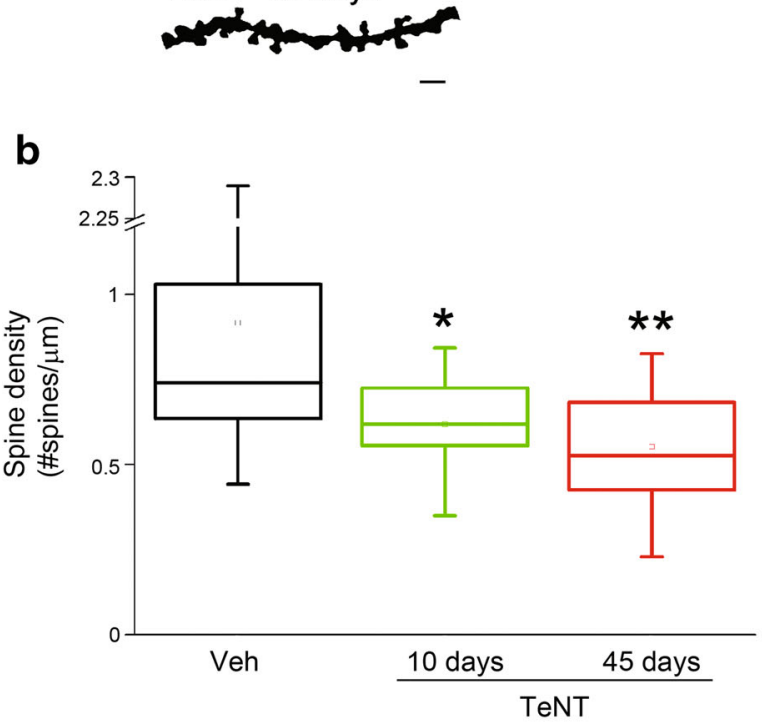

C

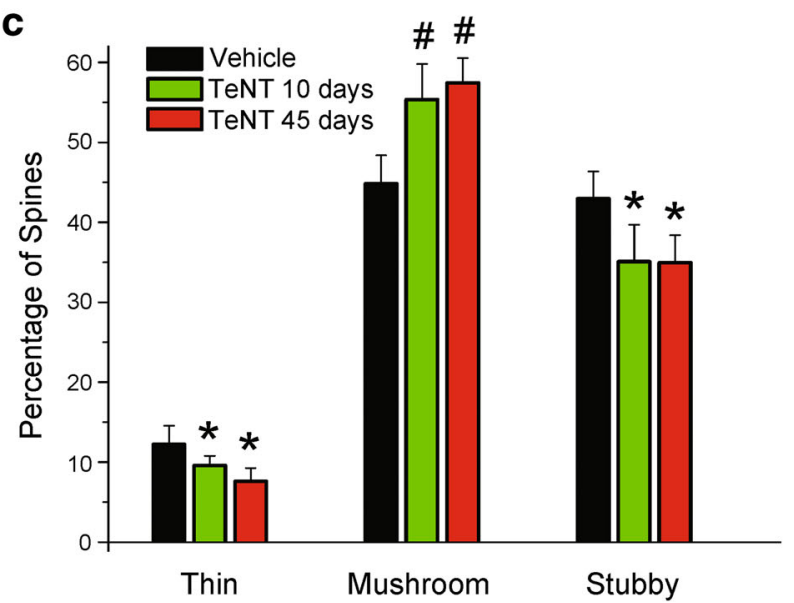

higher than $70 \%$ decreased from $47.7 \%$ in vehicle to $24.7 \%$ in TeNT (Two-sample Kolmogorov-Smirnov test, $p<0.05$, Fig. 5d). We conclude that the fraction of responsive units is reduced in the chronically epileptic cortex. Interestingly, however, if we compare the peak-tobaseline ratios using only the set of responsive units for vehicle and TeNT animals, the difference is still significant $(p<0.05, t$ test). So both a decrease in the number of responsive units and a reduction in their sensitivity occur in TeNT mice.
4Fig. 4 Reduction of spine density in epileptic mice. a Representative images of a dendritic segment in vehicle (top), and TeNT-infused mice at 10 days (middle) and 45 days (bottom). Raw images have been similarly processed with Adobe Photoshop tools to enhance visibility of spines. Scale bar $2 \mu \mathrm{m}$. b Overall density of dendritic protrusions in vehicle (Veh) and TeNT-infused mice at 10 and 45 days. Neurons of epileptic animals show a consistent and highly significant decrease in the density of spines (one-way ANOVA on ranks $p<0.01$; followed by Dunn's test, $p<0.05$ for TeNT 10 days and 0.01 for TeNT 45 days). The horizontal lines in the box chart denote the 25 th, 50th, and 75 th percentile values. The error bars denote the 5th and 95th percentile values, while the square indicates the mean of the data. c Changes in the proportion of spine morphological subtypes in the epileptic mice. In both groups of TeNT-injected mice there is an increase in the proportion of mushroom spines and a decrease in the proportion of both thin and stubby ones (one-way ANOVA, $p<0.01$; followed by post hoc test: $p<0.01$ for mushroom; $p=0.02$ for thin; $p=0.02$ for stubby). ${ }^{*} p<0.05 ;{ }^{*} p<0.01$. Data are mean \pm SEM

The data described above suggest that the dampened peak-to-baseline ratio in neurons from chronically epileptic animals may be due to a reduced reliability of visual responses. Indeed, we found a higher incidence of failures (i.e., the proportion of light bar passages unable to modify spontaneous activity) in TeNT- vs. vehicle-treated mice ( $t$ test, $p<0.006$; Fig. 5e). We checked whether failure trials were characterized by higher spiking activity before stimulus presentation, with respect to trials with normal responses in the chronic TeNT group. Indeed we found that pre-stimulus firing rates were significantly higher when response failed (paired $t$ test, $p=0.008$ ) in TeNT-treated mice at 45 days (Fig. 5f). These results indicate that spontaneous, stimulus-independent activation of the hyperexcitable network substantially interferes with visually-evoked firing of cortical units in the epileptic cortex.

\section{Contrast response function in epileptic animals}

We used VEP recordings to further characterize visual responsiveness in chronically epileptic mice (45 days after TeNT). We found a significant reduction of absolute VEP amplitudes in TeNT-treated vs. vehicle-infused mice ( $t$ test, $p<0.05$; Fig. 6a). Latency of visual drive (i.e. of the major positive VEP peak) was unaltered by treatment (Mann-Whitney rank sum test, $p=0.465$; data not shown). Similar to the spiking activity, we also found a reduction in the signal-to-noise ratio, i.e. the ratio of VEPs evoked by $90 \%$ contrast gratings and by a blank screen in the TeNT-infused mice ( $t$ test, $p=0.007$; Fig. $6 \mathrm{~b}$ ). We also evaluated visual responses at different contrast levels and we found that visual responses in TeNT mice were depressed across a range of visual contrasts (two-way repeated measures ANOVA followed by Holm-Sidak test; $p<0.05$ for $20 \%$ and $30 \% ; p<0.001$ for $90 \%$; Fig. $6 c$ ). 

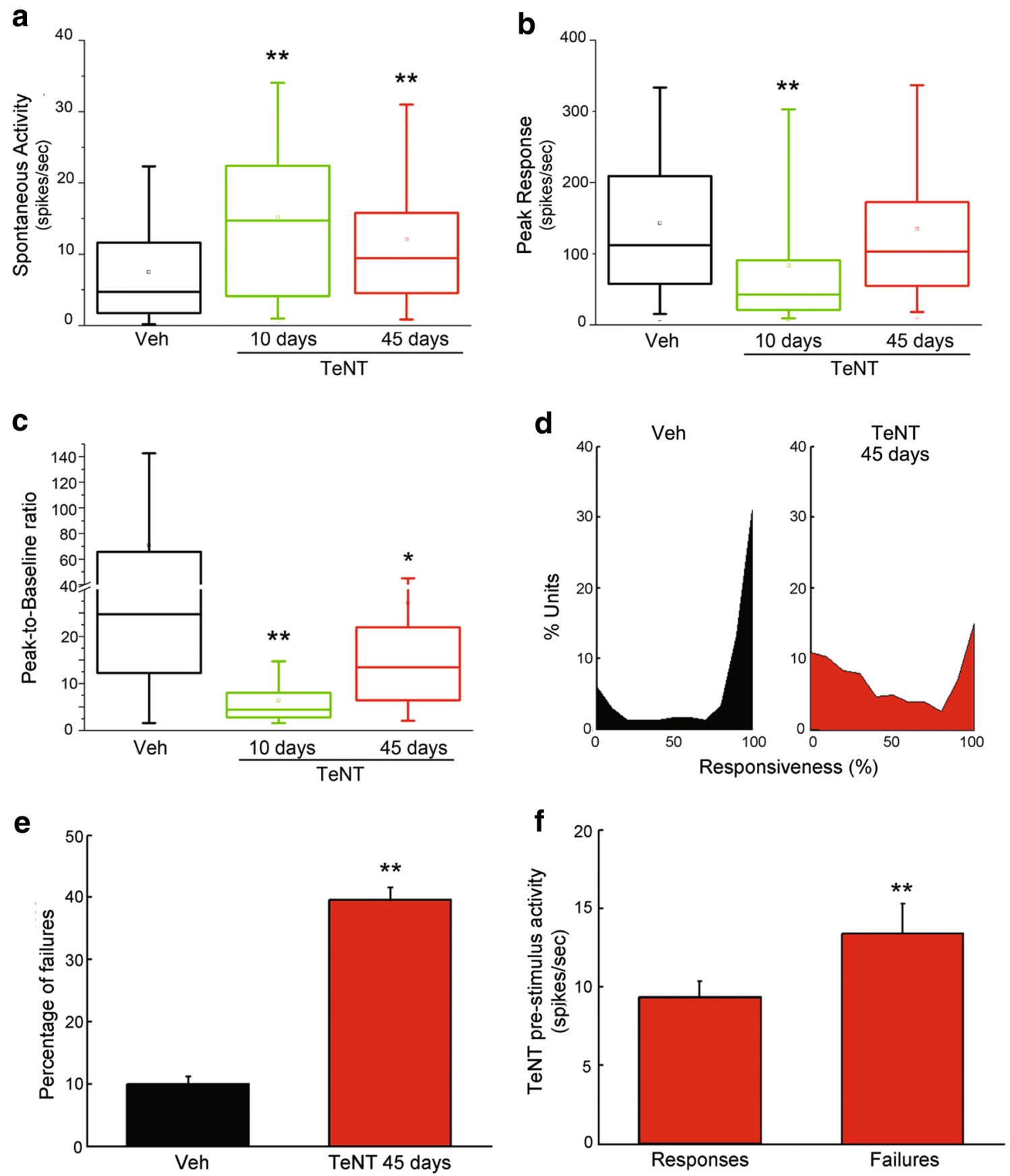

We next investigated if the changes in responsiveness measured as absolute value of the response in TeNT-injected mice were associated to a different shape of the response function. We modelled the contrast-response function as standard hyperbolic ratio function separately for each animal as shown in Fig. 6d (Albrecht and Hamilton 1982; Contreras and Palmer 2003). Then, following the analysis performed in Vaiceliunaite et al. (2013), we compared the parameters indicating the sensitivity of the response, i.e., the semisaturation

contrast and the response exponent, in vehicle- and TeNT-injected mice (see Methods for details). We found that the semisaturation contrast was not significantly different in the two conditions (unpaired $t$ test, $p>0.7,26.5 \pm 7 \%$ in Veh, $28 \pm 7 \%$ in TeNT) and the same was true for the exponent (unpaired $t$ test, $p>0.75,2.3 \pm 0.6$ in Veh, $2.5 \pm 1$ in TeNT). These results overall indicate that following TeNT injection the VEP contrast response function is rescaled but its shape is not affected. 
4Fig. 5 Increased spontaneous discharge and reduced responsiveness to visual stimuli in the TeNT-treated cortex. a Quantification of spontaneous firing rates of cortical units in the vehicle-injected cortex (Veh) and in the TeNT-treated cortex (TeNT), 10 and 45 days after toxin delivery. Note the significant increase of spontaneous discharge in both TeNT-injected groups (one-way ANOVA on ranks, $p<0.001$; followed by Dunn's test, $p<0.01)$. b Quantification of peak response of cortical units in vehicle- (Veh) and in TeNT-treated cortex (TeNT), 10 and 45 days after toxin injection. TeNT mice at 10 days display a decrease in firing rates following visual stimulation, which is recovered at 45 days (one-way ANOVA on ranks, $p<0.001$; followed by Dunn's test $p<0.01$ for TeNT 10 days). c Visual responsiveness of cortical units calculated as the ratio between peak discharge following visual stimulation and spontaneous activity. In both TeNT-injected groups the visual responsiveness is decreased (one-way ANOVA on ranks, $p<0.001$; followed by Dunn's test, $p<0.01$ for TeNT 10 days and $p<0.05$ for TeNT 45 days). In panels $\mathrm{a}, \mathrm{b}$ and $\mathrm{c}$, data are displayed as box chart plots. The horizontal lines in each box denote the 25th, 50th, and 75th percentile values. The error bars denote the 5th and 95th percentile values. The square symbols denote the mean of the column of data. Number of recorded cells are as follows: Veh: 183 cells from 11 animals; TeNT 10 days: 120 cells from 5 animals; TeNT 45 days: 161 cells from 10 animals. d Percentage frequency distribution of responsiveness for units recorded in vehicle (left) and TeNT mice at 45 days (right). The two distributions are significantly different (Twosample Kolmogorov-Smirnov test, $p<0.05$, Veh $n=3$, TeNT 45 days $n=7$ ) e Percentage of failures (i.e. lack of response to a light bar drifting into the receptive field) of cortical units. The reduced responses in TeNT animals are due to a high percentage of failures ( $t$ test, $p=0.001$; Veh $n=3$, TeNT 45 days $n=7$ ). f Analysis of pre-stimulus activity in TeNT group. Failure trials are preceded by higher spiking activity (paired $t$ test, $p=0.008$, TeNT 45 days $n=7$ )

\section{Reduced spatial resolution (acuity) of the epileptic cortical network}

Spatial resolution is a fundamental property of primary visual cortex and underlies the ability to see fine detail. To ascertain whether visual acuity was affected in adult epileptic mice, we recorded VEPs elicited across a series of spatial frequencies of the stimulus grating in vehicle- and TeNT-treated mice at 45 days, in the injected hemisphere. The data showed that VEP visual acuity was consistently lower in epileptic animals as compared to controls ( $t$ test, $p<0.001$; Fig. 7a). Interestingly, VEP visual acuity was similarly impaired also in the contralateral, untreated hemisphere of TeNT-injected mice $(n=4$; one-way ANOVA followed by Holm-Sidak, $p=0.001$ for Veh vs TeNT contralateral; $p=0.37$ for TeNT ipsilateral vs TeNT contralateral; data not shown).

To examine whether overall visual abilities were affected also at the behavioral level in TeNT-infused mice, we tested the animals in a classical two-alternative forcedchoice visual discrimination task, the Prusky water box (Prusky et al. 2000). In this task, animals learn to associate a stimulus grating with the presence of a submerged platform. We found that epileptic mice were significantly slower in task acquisition, as demonstrated by the increase in the number of sessions required to reach criterion ( $t$ test, $p<0.01$; Fig. $7 \mathrm{~b}$ ). Interestingly, only two-thirds (6 out of 9) of the TeNT animals were able to learn the task, while all the vehicle mice ( 7 out of 7 ) did so. Behavioral visual acuity (i.e. the highest spatial frequency that could be discriminated) was measured only in the animals that effectively learned the task. The results showed that behavioral acuity was significantly lower in TeNT-injected mice as compared to controls (Veh, $t$ test, $p<0.05$; Fig. 7c). Altogether, these data demonstrate robust impairments in sensory processing in epileptic mice.

\section{Discussion}

TeNT injection has been previously used to induce focal epilepsy in the brain (Jefferys and Walker 2006). Delivery of TeNT into the hippocampus provides a model of temporal lobe epilepsy (Ferecsko et al. 2014; Jiruska et al. 2010), while injection into the motor cortex results in focal neocortical epilepsy resembling epilepsia partialis continua (Louis et al. 1990; Nilsen et al. 2005). TeNT-induced chronic epilepsy lacks an initial status epilepticus and is accompanied by minimal or no neuronal loss (Jefferys and Walker 2006; Mainardi et al. 2012). Thus, the epileptic syndrome likely depends on alterations in synaptic function and imbalances in inhibitory vs. excitatory neurotransmission, making the TeNT model an ideal system to investigate the mechanisms at the basis of seizure generation in non-lesional epilepsy. TeNT-induced seizures are also resistant to conventional anti-epileptic drugs (Nilsen et al. 2005), suggesting the potential of this model for drug screening.

While most of the previous work on TeNT-induced epilepsy was carried out in the hippocampus or motor cortex, here we have exploited TeNT delivery into the visual cortex to measure quantitatively alterations in sensory processing in the epileptic zone and the biochemical and anatomical underpinnings of cortical dysfunction. Specifically, we measured visual responses together with biochemical and morphological changes at the peak of TeNT proteolytic action (10 days) and at the completion of toxin effects (45 days). This allowed us to determine those circuit alterations that outlast toxin activity and may be at the basis of the persistent epileptic phenotype.

We outline here a possible series of events that link functional observations with the underlying biochemical/ structural findings. In the acute phase immediately after injection, TeNT acts selectively on presynaptic terminals by cleaving the synaptic vesicle protein VAMP (Rossetto et al. 2013). Ferecsko et al. (2014) have clearly shown that TeNT dramatically reduces GABAergic inhibition 

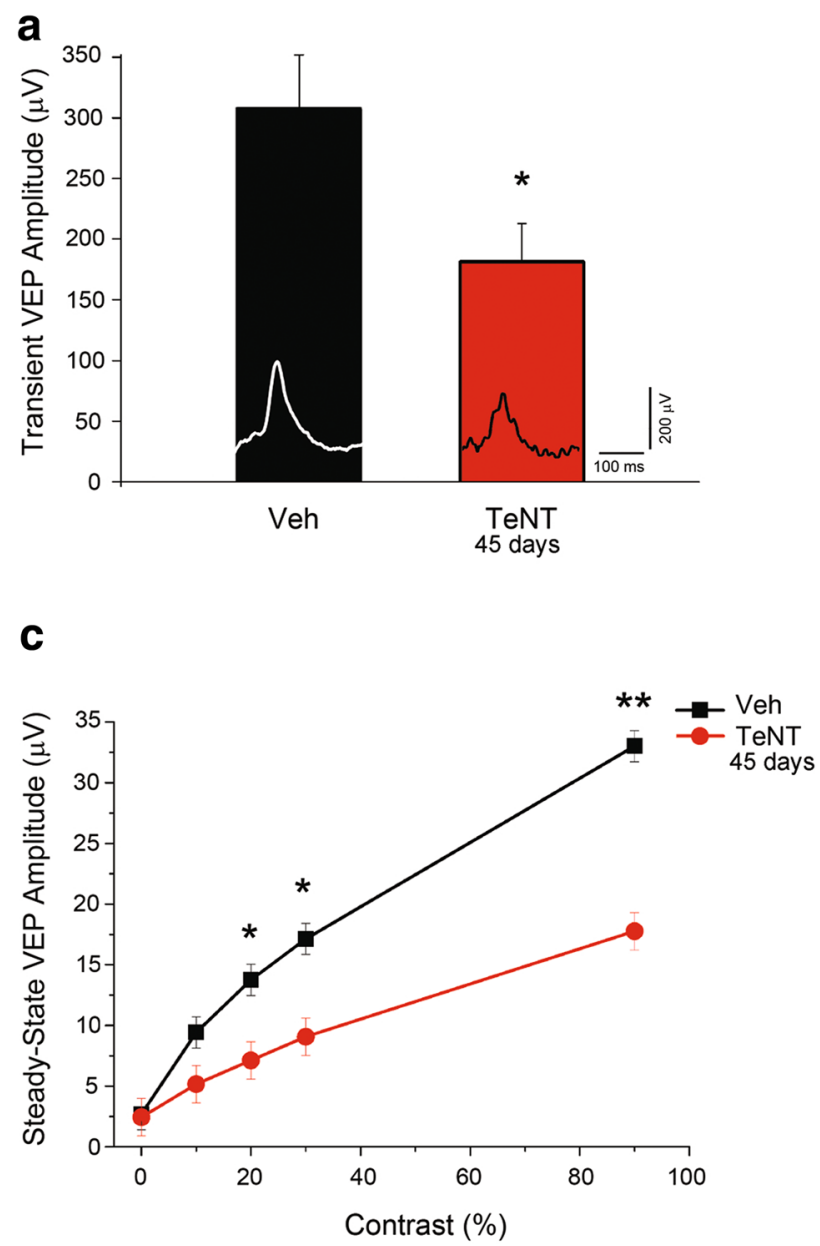
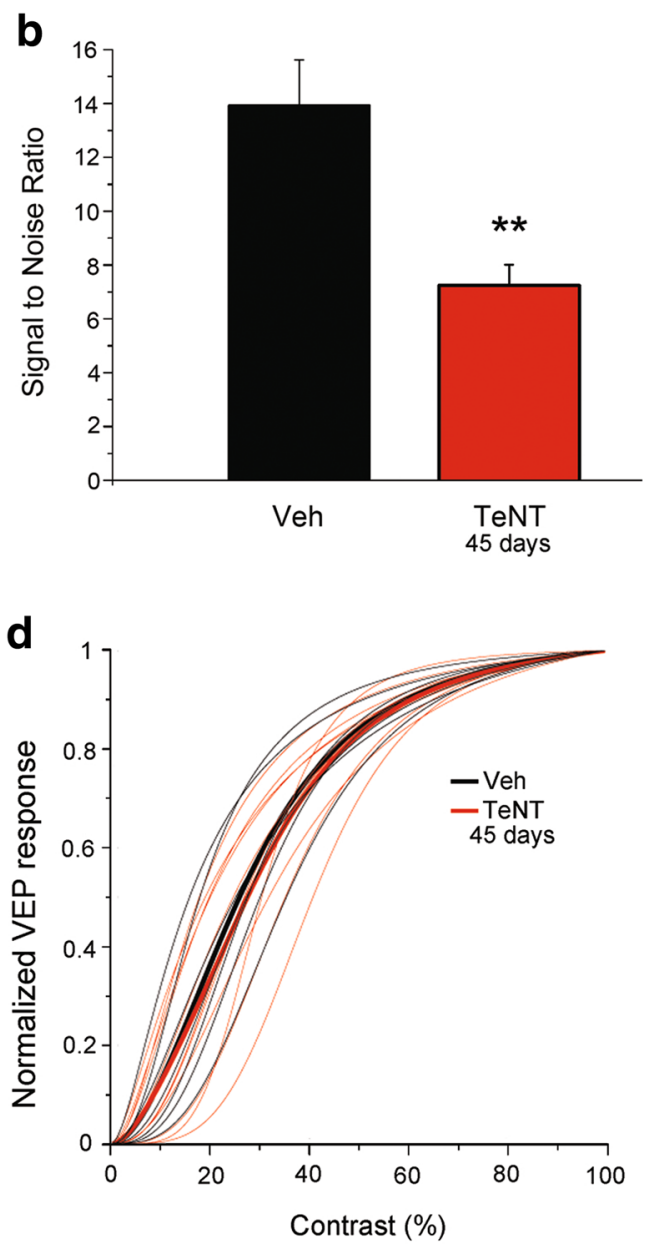

Fig. 6 Reduced VEP responses and altered cortical gain control in epileptic mice. All recordings were performed starting from 45 days following cortical injection. a Transient VEPs amplitude in TeNTand Vehicle-injected mice. Representative VEPs responses for each group are shown inside the histograms. Responses in TeNT-injected mice are consistently lower compared to control ( $t$ test $p<0.05$; Veh $n=17$, TeNT $n=16$ ). Visual stimulus: square wave grating alternating at $1 \mathrm{~Hz}$, spatial frequency $0.06 \mathrm{c} / \mathrm{deg}$, contrast $90 \%$. b Responsiveness of visual cortex in vehicle and TeNT-treated mice, measured as the ratio between VEPs amplitude under optimal stimulus conditions (sinusoidal grating alternating at $4 \mathrm{~Hz}$, spatial frequency $0.06 \mathrm{c} / \mathrm{deg}$, contrast $90 \%$ ) and the VEP response to a blank stimulus. Note the lower ratio in TeNT-injected mice ( $t$ test,

but has also effect on excitatory neurotransmission. This double effect may explain the induction of epileptiform activity (via silencing of inhibitory inputs) and also the dampening of visual responsiveness (via downregulation of excitatory transmission) at 10 days. Interestingly, VAMP2 levels were strongly reduced at 10 days and did not recover completely in the chronic stage (Fig. 1b). This could indicate a persistent alteration in neurotransmission that maintains hyperexcitability and reduced visual responses even when the toxin has been cleared from the system. At the structural level, epileptiform, high-frequency activity in the acute phase may on one $p=0.007 ;$ Veh $n=11$, TeNT $n=7)$. c The amplitude of steadystate VEPs is plotted as a function of variable contrast $(0,10,20,30$, $90 \%$ ) in control and TeNT-injected mice. VEPs responses are strongly reduced in TeNT visual cortex with respect to Vehicle (twoway repeated measures ANOVA followed by Holm-Sidak test; $p<0.05$ for 20 and $30 \%$; $p<0.001$ for $90 \%$ ). Visual stimulus: sinusoidal grating alternating at $4 \mathrm{~Hz}$, spatial frequency $0.06 \mathrm{c} / \mathrm{deg}$, contrast $0,10,20,30,90 \%$; Veh $n=11$, TeNT $n=7$. d Normalized hyperbolic ratio functions fitting VEP response contrast modulation in different TeNT- (red) and Vehicle- (black) injected mice. Bold lines represent averages over all animals for each group (Veh $n=11$, TeNT $n=7$ )

hand damage the postsynaptic compartment (resulting in "dendrotoxicity" and reduced spine densities, Swann et al. 2000; Wong and Guo 2013), and on the other hand trigger the conversion of a subset of thin spines into mushroom spines (Bourne and Harris 2007). This structural trace (i.e. higher proportion of mushroom spines) persists in the chronic stage and may be responsible for the higher frequency of spontaneous discharge in TeNTtreated cortical neurons, and for the increased incidence of failures (due to stimulus-independent activation of the hyperexcitable network that occludes visually-evoked responses). 


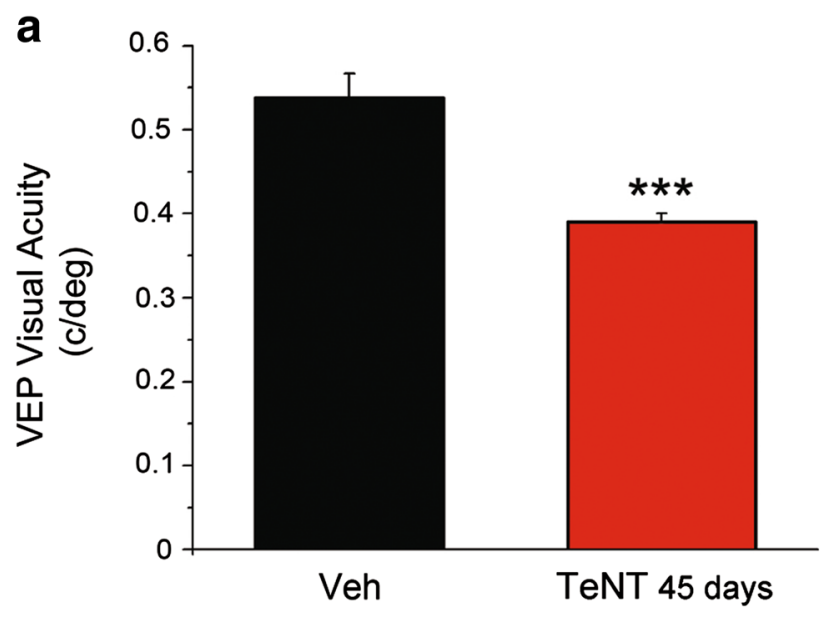

b

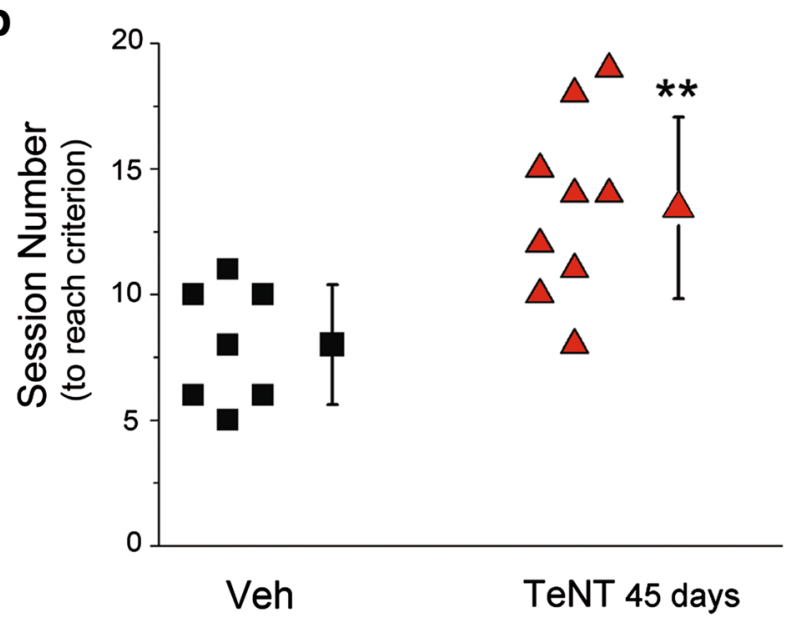

C

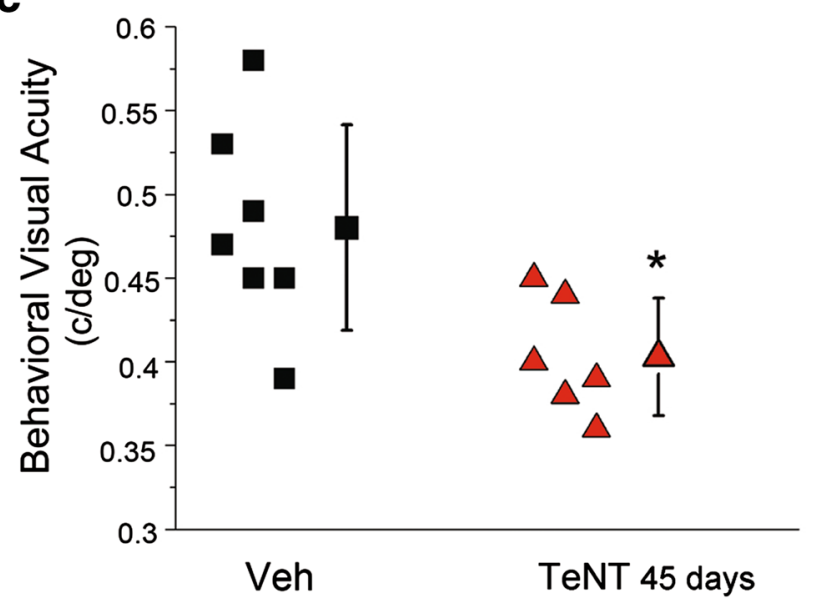

This scenario is based on correlative evidence, but it has been generally very difficult to demonstrate causal links between structural and functional plasticity in the current literature (Djurisic et al. 2013; Hofer et al. 2009; Holtmaat and Svoboda 2009; Miquelajauregui et al. 2015; Tropea et al. 2010). Despite the fact that the TeNT model is amply
4Fig. 7 Impaired electrophysiological and behavioral visual acuity in epileptic mice. a Electrophysiological assessment of visual acuity in vehicle and TeNT-injected mice. Visual acuity, assessed 45 days post injection, is significantly reduced in TeNT-injected mice ( $t$ test, $p<0.001$; Veh $n=5$, TeNT $n=6$ ). b Quantification of the number of training sessions required to reach criterion (80\% correct choices) for vehicle and TeNT-injected mice in the Prusky water box. Epileptic mice took significantly longer to acquire the task ( $t$ test, $p<0.01$; Veh $n=7$, TeNT $n=9$ ). c Summary of data on behavioral visual acuity. Each symbol represents one animal, and only animals that reached criterion were tested for spatial resolution ( 6 out of 9 in TeNT group, 7 out of 7 in vehicle). Note the reduced visual acuity in TeNT-injected animals compared to vehicle ( $t$ test, $p<0.05$; Veh $n=7$, TeNT $n=6$ )

employed, there is yet no consensus on the precise processes that lead to a permanent hyperexcitable phenotype. The present data significantly add to our understanding of the cortical rearrangements by which TeNT induces a persistent epileptic focus.

\section{Alterations in sensory processing in the TeNT- injected cortex}

The data reported in this manuscript demonstrate substantial alterations in spontaneous activity and sensory responses in focal neocortical epilepsy induced by TeNT. Specifically, we found that baseline discharge was increased, whereas visual responses were less reliable in the epileptic cortex. Alterations in visual responses were evident both at the level of VEPs (that measure the integrated subthreshold activity of a population of cortical neurons; (Porciatti et al. 1999) and at the level of spiking activity of cortical units. We used an anesthetized mouse preparation that is not suited to the evaluation of epileptiform activity (due to the interfering effect of anesthesia) but is ideal for mapping visual responses. Thus, the aim was to monitor baseline changes of hyperexcitable circuits that lead to dysfunction of the cortical network and impaired sensory processing (Grant 2005).

Recordings of spiking activity showed a clear enhancement of spontaneous discharge of cortical neurons. This was evident well beyond the time window of TeNT action, suggesting that intrinsic excitability of cortical neurons is persistently modified in the epileptic cortex (Wykes et al. 2012). Visual responsiveness was also persistently affected. In particular, we noted a higher incidence of failures in responding to visual stimuli in the chronically epileptic cortex. Interestingly, trials with failures were preceded by higher spontaneous activity than those with normal responses. In other words, TeNT treatment appears to increase firing rates in baseline, unstimulated conditions and to reduce the probability of activating visually-evoked discharges. Altogether these findings 
suggest that spontaneous, stimulus-independent activation of the hyperexcitable network occludes visually evoked depolarization and firing of cortical units.

In order to elucidate the cell identity of the recorded units, we performed a spike waveform analysis (Mitchell et al. 2007) in both vehicle and TeNT animals. We examined the distribution of spike waveform widths and we found a unimodal distribution with a median around $0.6 \mathrm{~ms}$ (data not shown). This result suggests that we recorded only from one class of neurons which, based on the broad median waveform width and the characteristics of our electrode, likely correspond to pyramidal cells (Mitchell et al. 2007). Further studies are needed to characterize the patterns of spontaneous and evoked discharge of inhibitory neurons within the TeNT-injected cortex.

Our data are consistent with previous observations of altered responses to sensory stimuli in focal epilepsy. In temporal lobe epilepsy, there is evidence for a reduction of auditory event-related potentials ipsilateral to the epileptogenic zone (Abubakr and Wambacq 2003). Particularly relevant for the present study, functional magnetic resonance imaging has revealed abnormalities in visual activation maps following stimulation with alternating checkerboard patterns in patients with occipital lobe epilepsy (Masuoka et al. 1999). In these subjects, the abnormal response to visual stimuli may be used for the presurgical localization of the epileptogenic side (Masuoka et al. 1999). Overall, the presence of specific perceptual disturbances in focal epilepsy syndromes is well documented, with most of the studies pointing to impaired sensory processing (Grant 2005). These data suggest that epileptogenic alterations in cortical networks may not only predispose to seizures and sudden bursts of activity, but also interfere substantially with cortical function even during inter-ictal periods.

Importantly, we documented a consistent reduction of visual acuity in TeNT-injected mice. This was evident at both the electrophysiological and behavioral level, with epileptic mice taking longer to learn a visual discrimination task and displaying poorer spatial resolution. Visual acuity can be significantly impaired by manipulations of visual experience during early development (Fagiolini et al. 1994), even when activity in primary visual cortex is manipulated unilaterally (Caleo et al. 2007). Conversely, acuity is much more resilient to changes in sensory input during adulthood (Baroncelli et al. 2012). It is well known that the developmental maturation of visual acuity in the occipital cortex depends on maturation of GABAergic neurotransmission (Huang et al. 1999) and that inhibitory connections sculpt receptive fields of cortical neurons (Harauzov et al. 2010; Ramoa et al. 1988). In this context, the dramatic impact of TeNT on GABAergic neurotransmission (Ferecsko et al. 2014) may lead to a persistently altered sampling of the visual field, and consequent loss of spatial resolution in the epileptic cortex.

\section{Potential substrates of long-term hyperexcitability}

In TeNT-induced cortical hyperexcitability, epileptiform alterations are detectable well beyond the time window of toxin action, indicating a reorganization of cortical circuits that leads to a permanent epileptic state. It is of great interest to determine the epileptogenic changes that leave a lasting trace in cortical excitability. In this context, we have shown a long-term depletion of the TeNT substrate VAMP2 and upregulation of GABAergic markers such as GAD65/67. GAD67 represents the major biosynthetic enzyme for GABA while GAD65 is expressed at the synapse to provide additional GABA release during intense activity (Erlander et al. 1991; Reetz et al. 1991). In keeping with the present results, upregulation of GAD mRNA was previously reported in the TeNT-infused rat hippocampus (Najlerahim et al. 1992). The enhancements in GABA biosynthetic enzymes may be interpreted as adaptive or homeostatic phenomena that tend to compensate for the hyperexcitability phenotype. Similar compensatory changes have been described in other models of epilepsy. For example, following limbic seizures granule cells express high levels of GAD67, likely in the attempt to limit seizure occurrence (Schwarzer and Sperk 1995). However, increased GABA signaling may also play a paradoxical role in synchronizing neuronal networks and promoting epileptiform activity (Avoli and de Curtis 2011).

We have also found striking rearrangements in dendritic structure of pyramidal neurons in the TeNT-treated cortex. A reduction in spine density in TeNT-treated neurons was already apparent 10 days after the toxin and persisted up to 45 days. Dendritic spines represent the major site of contact for excitatory inputs to cortical neurons and play a key role in the control of neuronal excitability. They are highly motile and dynamic even in the adult cerebral cortex (Holtmaat and Svoboda 2009). Reductions in spine densities have been found in other models of epilepsy [(Colciaghi et al. 2014; Singh et al. 2013; Swann et al. 2000); for a review see (Wong and Guo 2013)], but the physiological significance of such changes is still debated. Spine loss could be a byproduct of intense seizure activity that damages the postsynaptic compartment ("dendrotoxicity"), or result from excessive synaptic pruning due to enhanced activity and synaptic competition within the epileptic focus (Swann et al. 2000; Wong and Guo 2013).

Interestingly, despite the overall reduction in spine density, an analysis of the proportion of morphological spine types revealed that mushroom spines were significantly preserved in TeNT-treated neurons at the expense of thin, immature protrusions. To our knowledge, this is the 
first description of a redistribution of spine types in epileptic brain tissue.

Mushroom spines are considered stable and "mature" contacts with a large head (Bosch and Hayashi 2012). Interestingly, high frequency activity leads to an enlargement of thin spines into mushroom spines (Bourne and Harris 2007). This finding suggests that sustained activity (such as that occurring during epileptic discharges) may triggers the "conversion" of small, thin spines into mature, enlarged protrusions. Since spine volume is positively correlated with synaptic strength, a preservation of mushroom spines may maintain overall excitatory drive onto cortical cells. Overall, excitatory input onto pyramidal neurons may even be increased in the chronic epileptic focus, taking into account the increased dendritic length of neurons in the TeNT-treated animals at 45 days (see Fig. 3c). Complexity of the dendritic tree (as shown by Sholl analysis) was also persistently modified by TeNT. Modelling work has clearly shown that dendritic morphology is an important determinant of firing patterns in neocortical pyramidal neurons (van Elburg and van Ooyen 2010), and may therefore impact on both seizure generation and baseline information processing.

In summary, we have described long-term alterations in spontaneous discharge and sensory responses within a TeNT-induced neocortical epilepsy focus. These functional modifications are accompanied by significant restructuring of the dendritic arbors of pyramidal neurons and by an upregulation of GABAergic markers. These changes likely represent the underlying substrate of hyperexcitability and deficits in information processing within the epileptic focus.

Acknowledgments The authors thank Francesca Biondi for invaluable technical support. This work was supported by a grant from Fondazione Pisa (to M.C.) and by Italian Ministry of Research (FIRB2010-RBFR10ZBYZ_003) to L.R. The funders had no role in study design, data collection and analysis, decision to publish, or preparation of the manuscript.

\section{References}

Abubakr A, Wambacq I (2003) The localizing value of auditory event-related potentials $(\mathrm{P} 300)$ in patients with medically intractable temporal lobe epilepsy. Epilepsy Behav 4:692-701 $\mathbf{S 1 5 2 5 5 0 5 0 0 3 0 0 2 3 3 6}$

Albrecht DG, Hamilton DB (1982) Striate cortex of monkey and cat: contrast response function. J Neurophysiol 48:217-237

Antonucci F, Bozzi Y, Caleo M (2009) Intrahippocampal infusion of botulinum neurotoxin E (BoNT/E) reduces spontaneous recurrent seizures in a mouse model of mesial temporal lobe epilepsy. Epilepsia 50:963-966. doi:10.1111/j.1528-1167.2008.01983

Avoli M, de Curtis M (2011) GABAergic synchronization in the limbic system and its role in the generation of epileptiform activity. Prog Neurobiol 95:104-132. doi:10.1016/j.pneurobio. 2011.07.003
Baldini S, Restani L, Baroncelli L, Coltelli M, Franco R, Cenni MC, Maffei L, Berardi N (2013) Enriched early life experiences reduce adult anxiety-like behavior in rats: a role for insulin-like growth factor 1. J Neurosci 33:11715-11723. doi:10.1523/ JNEUROSCI.3541-12.2013

Baroncelli L, Bonaccorsi J, Milanese M, Bonifacino T, Giribaldi F, Manno I, Cenni MC, Berardi N, Bonanno G, Maffei L, Sale A (2012) Enriched experience and recovery from amblyopia in adult rats: impact of motor, social and sensory components. Neuropharmacology 62:2388-2397

Bosch M, Hayashi Y (2012) Structural plasticity of dendritic spines. Curr Opin Neurobiol 22:383-388. doi:10.1016/j.conb.2011.09. 002

Bourne J, Harris KM (2007) Do thin spines learn to be mushroom spines that remember? Curr Opin Neurobiol 17:381-386. doi:10. 1016/j.conb.2007.04.009

Caleo M, Medini P, von Bartheld CS, Maffei L (2003) Provision of brain-derived neurotrophic factor via anterograde transport from the eye preserves the physiological responses of axotomized geniculate neurons. J Neurosci 23:287-296 23/1/287

Caleo M, Restani L, Gianfranceschi L, Costantin L, Rossi C, Rossetto O, Montecucco C, Maffei L (2007) Transient synaptic silencing of developing striate cortex has persistent effects on visual function and plasticity. J Neurosci 27:4530-4540 27/17/ 453010.1523/JNEUROSCI.0772-07.2007

Cerri C, Restani L, Caleo M (2010) Callosal contribution to ocular dominance in rat primary visual cortex. Eur J Neurosci 32:1163-1169. doi:10.1111/j.1460-9568.2010.07363.x

Colciaghi F, Finardi A, Nobili P, Locatelli D, Spigolon G, Battaglia GS (2014) Progressive brain damage, synaptic reorganization and NMDA activation in a model of epileptogenic cortical dysplasia. PLoS One 9:e89898. doi:10.1371/journal.pone. 0089898

Contreras D, Palmer L (2003) Response to contrast of electrophysiologically defined cell classes in primary visual cortex. J Neurosci 23:6936-6945 23/17/6936

Corradini I, Donzelli A, Antonucci F, Welzl H, Loos M, Martucci R, De Astis S, Pattini L, Inverardi F, Wolfer D, Caleo M, Bozzi Y, Verderio C, Frassoni C, Braida D, Clerici M, Lipp HP, Sala M, Matteoli M (2014) Epileptiform activity and cognitive deficits in SNAP-25( \pm ) mice are normalized by antiepileptic drugs. Cereb Cortex 24:364-376. doi:10.1093/cercor/bhs316

Djurisic M, Vidal GS, Mann M, Aharon A, Kim T, Ferrao Santos A, Zuo Y, Hubener M, Shatz CJ (2013) PirB regulates a structural substrate for cortical plasticity. Proc Natl Acad Sci USA 110:20771-20776. doi:10.1073/pnas.1321092110

Dudek FE, Sutula TP (2007) Epileptogenesis in the dentate gyrus: a critical perspective. Prog Brain Res 163:755-773. doi:10.1016/ S0079-6123(07)63041-6

Erlander MG, Tillakaratne NJ, Feldblum S, Patel N, Tobin AJ (1991) Two genes encode distinct glutamate decarboxylases. Neuron 7:91-100 0896-6273(91)90077-D

Fagiolini M, Pizzorusso T, Berardi N, Domenici L, Maffei L (1994) Functional postnatal development of the rat primary visual cortex and the role of visual experience: dark rearing and monocular deprivation. Vision Res 34:709-720 00426989(94)90210-0

Farisello P, Boido D, Nieus T, Medrihan L, Cesca F, Valtorta F, Baldelli P, Benfenati F (2013) Synaptic and extrasynaptic origin of the excitation/inhibition imbalance in the hippocampus of synapsin I/II/III knockout mice. Cereb Cortex 23:581-593. doi:10.1093/cercor/bhs041

Ferecsko AS, Jiruska P, Foss L, Powell AD, Chang WC, Sik A, Jefferys JG (2014) Structural and functional substrates of tetanus toxin in an animal model of temporal lobe epilepsy. Brain Struct Funct. doi:10.1007/s00429-013-0697-1 
Ferrari E, Gu C, Niranjan D, Restani L, Rasetti-Escargueil C, Obara I, Geranton SM, Arsenault J, Goetze TA, Harper CB, Nguyen TH, Maywood E, O'Brien J, Schiavo G, Wheeler DW, Meunier FA, Hastings M, Edwardson JM, Sesardic D, Caleo M, Hunt SP, Davletov B (2013) Synthetic self-assembling clostridial chimera for modulation of sensory functions. Bioconjug Chem 24:1750-1759. doi: $10.1021 / \mathrm{bc} 4003103$

Friedberg MH, Lee SM, Ebner FF (1999) Modulation of receptive field properties of thalamic somatosensory neurons by the depth of anesthesia. J Neurophysiol 81:2243-2252

Gianfranceschi L, Siciliano R, Walls J, Morales B, Kirkwood A, Huang ZJ, Tonegawa S, Maffei L (2003) Visual cortex is rescued from the effects of dark rearing by overexpression of BDNF. Proc Natl Acad Sci USA 100(12486-12491):1934. doi:10.1073/ pnas. 1934836100836100

Gibb R, Kolb B (1998) A method for vibratome sectioning of GolgiCox stained whole rat brain. J Neurosci Methods 79:1-4 S0165027097001635

Goel A, Lee HK (2007) Persistence of experience-induced homeostatic synaptic plasticity through adulthood in superficial layers of mouse visual cortex. J Neurosci 27:6692-6700. doi:10.1523/ JNEUROSCI.5038-06.2007

Grant AC (2005) Interictal perceptual function in epilepsy. Epilepsy Behav 6:511-519. doi:10.1016/j.yebeh.2005.03.016

Hagemann G, Hoeller M, Bruehl C, Lutzenburg M, Witte OW (1999) Effects of tetanus toxin on functional inhibition after injection in separate cortical areas in rat. Brain Res 818:127-134 S00068993(98)01293-1

Harauzov A, Spolidoro M, DiCristo G, De Pasquale R, Cancedda L, Pizzorusso T, Viegi A, Berardi N, Maffei L (2010) Reducing intracortical inhibition in the adult visual cortex promotes ocular dominance plasticity. J Neurosci 30:361-371. doi:10.1523/ JNEUROSCI.2233-09.2010

Hofer SB, Mrsic-Flogel TD, Bonhoeffer T, Hubener M (2009) Experience leaves a lasting structural trace in cortical circuits. Nature 457:313-317. doi:10.1038/nature07487

Holtmaat A, Svoboda K (2009) Experience-dependent structural synaptic plasticity in the mammalian brain. Nat Rev Neurosci 10:647-658. doi:10.1038/nrn2699

Huang ZJ, Kirkwood A, Pizzorusso T, Porciatti V, Morales B, Bear MF, Maffei L, Tonegawa S (1999) BDNF regulates the maturation of inhibition and the critical period of plasticity in mouse visual cortex Cell 98:739-755 S0092-8674(00)81509-3

Jefferys J, Walker M (2006) Tetanus toxin model of focal epilepsy. In: Pitkanen A, Schwartzkroin P, Moshé S (eds) Models of seizures and epilepsy. Elsevier Academic Press, Amsterdam, pp 407-414

Jiruska P, Finnerty GT, Powell AD, Lofti N, Cmejla R, Jefferys JG (2010) Epileptic high-frequency network activity in a model of non-lesional temporal lobe epilepsy. Brain 133:1380-1390. doi:10.1093/brain/awq070

Louis ED, Williamson PD, Darcey TM (1990) Chronic focal epilepsy induced by microinjection of tetanus toxin into the cat motor cortex. Electroencephalogr Clin Neurophysiol 75:548-557

Mainardi M, Landi S, Gianfranceschi L, Baldini S, De Pasquale R, Berardi N, Maffei L, Caleo M (2010) Environmental enrichment potentiates thalamocortical transmission and plasticity in the adult rat visual cortex. J Neurosci Res 88:3048-3059. doi:10. 1002/jnr.22461

Mainardi M, Pietrasanta M, Vannini E, Rossetto O, Caleo M (2012) Tetanus neurotoxin-induced epilepsy in mouse visual cortex. Epilepsia 53:e132-e136. doi:10.1111/j.1528

Marchetti C, Tafi E, Middei S, Rubinacci MA, Restivo L, AmmassariTeule M, Marie H (2010) Synaptic adaptations of CA1 pyramidal neurons induced by a highly effective combinational antidepressant therapy. Biol Psychiatry 67:146-154. doi:10. 1016/j.biopsych.2009.09.017
Masuoka LK, Anderson AW, Gore JC, McCarthy G, Spencer DD, Novotny EJ (1999) Functional magnetic resonance imaging identifies abnormal visual cortical function in patients with occipital lobe epilepsy. Epilepsia 40:1248-1253

Miquelajauregui A, Kribakaran S, Mostany R, Badaloni A, Consalez GG, Portera-Cailliau C (2015) Layer 4 pyramidal neurons exhibit robust dendritic spine plasticity in vivo after input deprivation. J Neurosci 35:7287-7294. doi:10.1523/JNEUR OSCI.5215-14.2015

Mitchell JF, Sundberg KA, Reynolds JH (2007) Differential attentiondependent response modulation across cell classes in macaque visual area V4 Neuron 55:131-141. doi:10.1016/j.neuron.2007. 06.018

Nahmani M, Erisir A (2005) VGluT2 immunochemistry identifies thalamocortical terminals in layer 4 of adult and developing visual cortex. J Comp Neurol 484:458-473. doi:10.1002/cne. 20505

Najlerahim A, Williams SF, Pearson RC, Jefferys JG (1992) Increased expression of GAD mRNA during the chronic epileptic syndrome due to intrahippocampal tetanus toxin. Exp Brain Res 90:332-342

Nilsen KE, Walker MC, Cock HR (2005) Characterization of the tetanus toxin model of refractory focal neocortical epilepsy in the rat. Epilepsia 46:179-187. doi:10.1111/j.0013-9580.2005. 26004.x

Pietrasanta M, Restani L, Cerri C, Olcese U, Medini P, Caleo M (2014) A switch from inter-ocular to inter-hemispheric suppression following monocular deprivation in the rat visual cortex Eur J Neurosci. doi:10.1111/ejn.12573

Pinto L, Drechsel D, Schmid MT, Ninkovic J, Irmler M, Brill MS, Restani L, Gianfranceschi L, Cerri C, Weber SN, Tarabykin V, Baer K, Guillemot F, Beckers J, Zecevic N, Dehay C, Caleo M, Schorle H, Gotz M (2009) AP2gamma regulates basal progenitor fate in a region- and layer-specific manner in the developing cortex. Nat Neurosci 12:1229-1237. doi:10.1038/nn.2399

Pitkanen A, Nehlig A, Brooks-Kayal AR, Dudek FE, Friedman D, Galanopoulou AS, Jensen FE, Kaminski RM, Kapur J, Klitgaard H, Loscher W, Mody I, Schmidt D (2013) Issues related to development of antiepileptogenic therapies. Epilepsia 54(Suppl 4):35-43. doi:10.1111/epi.12297

Pizzorusso T, Medini P, Landi S, Baldini S, Berardi N, Maffei L (2006) Structural and functional recovery from early monocular deprivation in adult rats. Proc Natl Acad Sci USA 103:8517-8522. doi:10.1073/pnas.0602657103

Porciatti V, Pizzorusso T, Maffei L (1999) The visual physiology of the wild type mouse determined with pattern VEPs. Vision Res 39:3071-3081 S0042-6989(99)00022-X

Prusky GT, West PW, Douglas RM (2000) Behavioral assessment of visual acuity in mice and rats. Vision Res 40:2201-2209 S00426989(00)00081-X

Ramoa AS, Paradiso MA, Freeman RD (1988) Blockade of intracortical inhibition in kitten striate cortex: effects on receptive field properties and associated loss of ocular dominance plasticity. Exp Brain Res 73:285-296

Reetz A, Solimena M, Matteoli M, Folli F, Takei K, De Camilli P (1991) GABA and pancreatic beta-cells: colocalization of glutamic acid decarboxylase (GAD) and GABA with synapticlike microvesicles suggests their role in GABA storage and secretion. EMBO J 10:1275-1284

Resta V, Novelli E, Vozzi G, Scarpa C, Caleo M, Ahluwalia A, Solini A, Santini E, Parisi V, Di Virgilio F, Galli-Resta L (2007) Acute retinal ganglion cell injury caused by intraocular pressure spikes is mediated by endogenous extracellular ATP. Eur J Neurosci 25:2741-2754. doi:10.1111/j.1460-9568.2007.05528.x

Restani L, Cerri C, Pietrasanta M, Gianfranceschi L, Maffei L, Caleo M (2009) Functional masking of deprived eye responses by 
callosal input during ocular dominance plasticity. Neuron 64:707-718. doi:10.1016/j.neuron.2009.10.019

Restani L, Antonucci F, Gianfranceschi L, Rossi C, Rossetto O, Caleo M (2011) Evidence for anterograde transport and transcytosis of botulinum neurotoxin A (BoNT/A). J Neurosci 31:15650-15659. doi:10.1523/JNEUROSCI.2618-11.2011

Restani L, Giribaldi F, Manich M, Bercsenyi K, Menendez G, Rossetto O, Caleo M, Schiavo G (2012) Botulinum neurotoxins $\mathrm{A}$ and $\mathrm{E}$ undergo retrograde axonal transport in primary motor neurons. PLoS Pathog 8:e1003087. doi:10.1371/journal.ppat. 1003087

Rossetto O, Scorzeto M, Megighian A, Montecucco C (2013) Tetanus neurotoxin. Toxicon 66:59-63. doi:10.1016/j.toxicon.2012.12. 027

Schiavo G, Matteoli M, Montecucco C (2000) Neurotoxins affecting neuroexocytosis. Physiol Rev 80:717-766

Schwarzer C, Sperk G (1995) Hippocampal granule cells express glutamic acid decarboxylase-67 after limbic seizures in the rat. Neuroscience 69:705-709 0306-4522(95)00348-M

Simonato M, Loscher W, Cole AJ, Dudek FE, Engel J Jr, Kaminski RM, Loeb JA, Scharfman H, Staley KJ, Velisek L, Klitgaard H (2012) Finding a better drug for epilepsy: preclinical screening strategies and experimental trial design. Epilepsia 53:1860-1867. doi:10.1111/j.1528-1167.2012.03541.x

Singh SP, He X, McNamara JO, Danzer SC (2013) Morphological changes among hippocampal dentate granule cells exposed to early kindling-epileptogenesis. Hippocampus 23:1309-1320. doi:10.1002/hipo.22169

Sorra KE, Harris KM (2000) Overview on the structure, composition, function, development, and plasticity of hippocampal dendritic spines. Hippocampus 10:501-511. doi:10.1002/10981063(2000)10:5<501:AID-HIPO1>3.0.CO;2-T

Swann JW, Al-Noori S, Jiang M, Lee CL (2000) Spine loss and other dendritic abnormalities in epilepsy. Hippocampus 10:617-625. doi:10.1002/1098-1063(2000)10:5<617:AID-HIPO13>3.0.CO;2-R

Tropea D, Majewska AK, Garcia R, Sur M (2010) Structural dynamics of synapses in vivo correlate with functional changes during experience-dependent plasticity in visual cortex. J Neurosci 30:11086-11095. doi:10.1523/JNEUROSCI.1661-10.2010

Vaiceliunaite A, Erisken S, Franzen F, Katzner S, Busse L (2013) Spatial integration in mouse primary visual cortex. J Neurophysiol 110:964-972. doi:10.1152/jn.00138.2013

van Elburg RA, van Ooyen A (2010) Impact of dendritic size and dendritic topology on burst firing in pyramidal cells. PLoS Comput Biol 6:e1000781. doi:10.1371/journal.pcbi.1000781

Whittington MA, Jefferys JG (1994) Epileptic activity outlasts disinhibition after intrahippocampal tetanus toxin in the rat. J Physiol 481(Pt 3):593-604

Wong M, Guo D (2013) Dendritic spine pathology in epilepsy: cause or consequence? Neuroscience 251:141-150. doi:10.1016/j. neuroscience.2012.03.048

Wykes RC, Heeroma JH, Mantoan L, Zheng K, MacDonald DC, Deisseroth K, Hashemi KS, Walker MC, Schorge S, Kullmann DM (2012) Optogenetic and potassium channel gene therapy in a rodent model of focal neocortical epilepsy. Sci Transl Med 4:161ra152. doi:10.1126/scitranslmed.3004190 